\title{
LABORATORY PROCEDURES
}

$\mathrm{U}-\mathrm{Pb}$ dating

$\mathrm{U}-\mathrm{Pb}$ dating was performed on the LA-ICPMS at Tokyo Institute of Technology with $\sim 20 \mu \mathrm{m}$ ablation pits. The ${ }^{207} \mathrm{~Pb} /{ }^{206} \mathrm{~Pb}$ ratio was corrected using NIST 610 standard reference material, and the ${ }^{206} \mathrm{~Pb} /{ }^{238} \mathrm{U}$ and ${ }^{232} \mathrm{Th} /{ }^{238} \mathrm{U}$ were corrected using reference zircon Nancy 91500 (Wiedenbeck et al., 1995). Analytical uncertainties combine the counting statistics and the 2 S.E. of standard analyses, added in quadrature. The common $\mathrm{Pb}$ correction assumed recent $\mathrm{Pb}$ loss and a common $\mathrm{Pb}$ composition given by the two-stage evolution model (Stacey and Kramers, 1975). No common Pb correction has been applied to analyses for which the corrected ratio is within 2 sigma error of the uncorrected ratio.

\section{Lu-Hf isotope analyses}

Lu-Hf isotope analyses were performed on the LA-MC-ICPMS at Tokyo Institute of Technology. Analyses were carried out with beam diameters of 35 or $63 \mu \mathrm{m}, 3-10 \mathrm{~Hz}$ repetition rates, and $\sim 60 \mathrm{sec}$ ablation times. Mass discrimination effects were corrected by normalizing to ${ }^{179} \mathrm{Hf} /{ }^{177} \mathrm{Hf}=0.7325$ (Patchett et al., 1981) for $\mathrm{Hf}$ and $\mathrm{Lu}$, and to ${ }^{173} \mathrm{Yb} /{ }^{171} \mathrm{Yb}=1.12346$ (Thirlwall and Anczkiewicz, 2004) for $\mathrm{Yb}$, using an exponential law. Interference of ${ }^{176} \mathrm{Lu}$ and ${ }^{176} \mathrm{Yb}$ onto ${ }^{176} \mathrm{Hf}$ was corrected by measuring ${ }^{176} \mathrm{Lu}$ and ${ }^{173} \mathrm{Yb}$ and using ${ }^{176} \mathrm{Lu} /{ }^{176} \mathrm{Lu}=0.026549$ (Chu et al., 2002) and ${ }^{176} \mathrm{Yb} /{ }^{173} \mathrm{Yb}=0.78696$ (Thirlwall and Anczkiewicz, 2004), respectively. For the calculation of initial Hf isotope ratio, the decay constant for ${ }^{176} \mathrm{Lu}$ proposed by Scherer et al. (2001) (1.865 x $\left.10^{-11} \mathrm{yr}^{-1}\right)$ was used.

\section{REFERENCE CITED}

Chu, N.C., Taylor, R.N., Chavagnac, V., Nesbitt, R.W., Boella, M., Milton, J.A., German, C.R., Bayon, G. and Burton, K., 2002. Hf isotope ratio analysis using multi-collector inductively coupled plasma mass spectrometry: an evaluation of isobaric intereference corrections: Jounal of Analytical Atomic Spectrometry, v. 17, p. $1567-1574$.

Patchett, P.J., Kuovo, O., Hedge, C.E. and Tatsumoto, M., 1981. Evolution of continental crust and mantle heterogeneity: evidence from $\mathrm{Hf}$ isotopes: Contribution to Mineralogy and Petrology, v. 78, p. 279-297.

Scherer, E., Münker, C. and Mezger, K., 2001. Calibration of the Lutetium-Hafnium 
Clock: Science, v. 293, p. 683-687.

Thirlwall, M. and Anczkiewicz, R., 2004. Multidynamic isotope ratio analysis using MC-ICP-MS and the causes of secular drift in $\mathrm{Hf}, \mathrm{Nd}$ and $\mathrm{Pb}$ isotope ratios: International Journal of Mass Spectrometry, v. 235, p. 59-81.

Stacey, J.S. and Kramers, J.D., 1975. Approximation of terrestrial lead isotope evolution by a two-stage model: Earth and Planetary Sciences Letters, v. 26, p. 207-221.

Wiedenbeck, M., Alle, P., Corfu, F., Griffin, W.L., Meier, M., Ober, F., Von Quant, A., Roddick, J.C. and Spiegel, J., 1995. Three natural zircon standards for U-Th-Pb, Lu-Hf, trace element and REE analyses: Geostandard Newsletter, v. 19, p. 1-23. 
lizuka et al. Fig. DR1

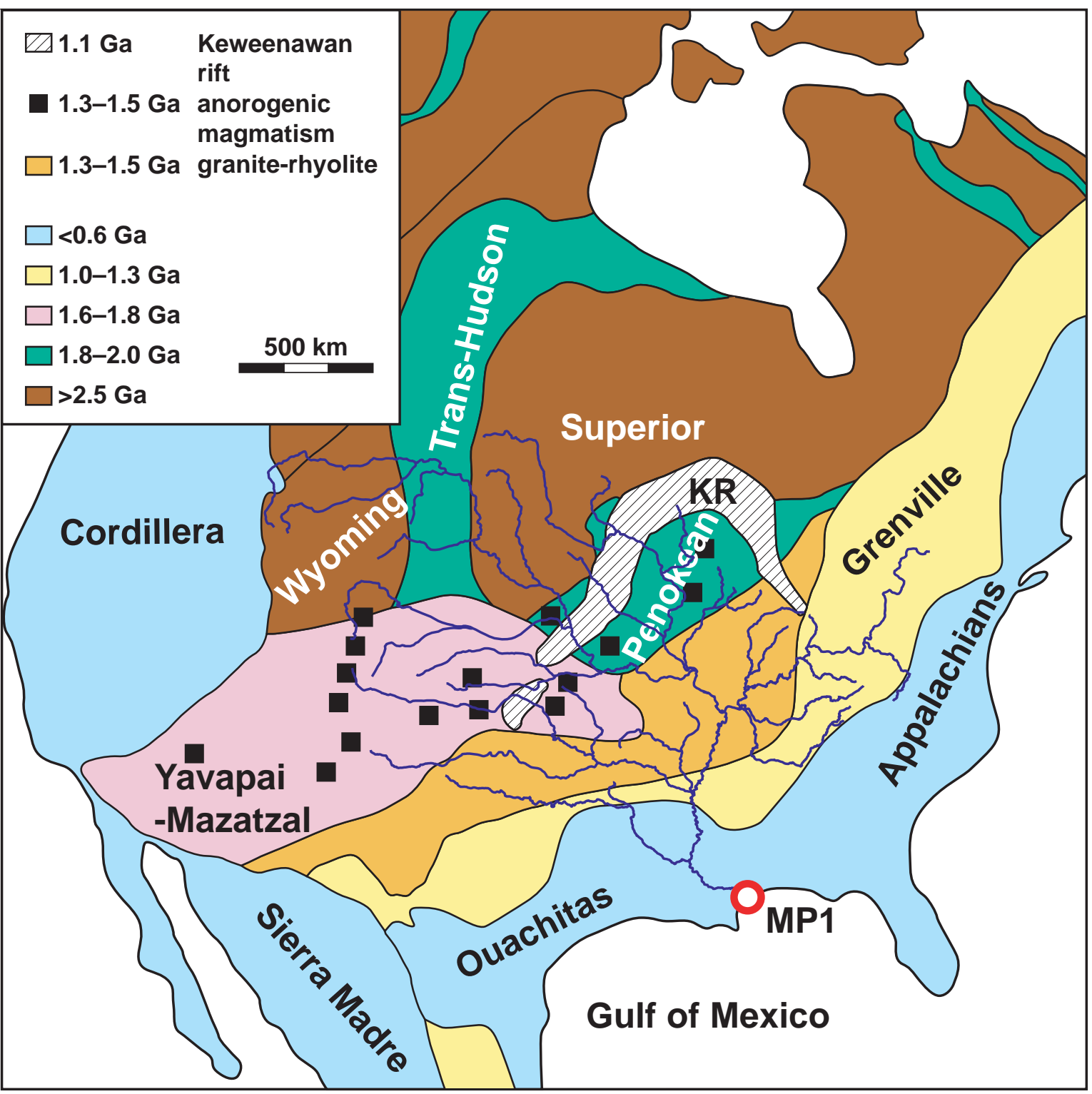

Figure DR1. Geologic map of basement in the North American continent (modified after Hoffman, 1989a; Rivers, 1997). Sample locality of MP1 (30²9'40”N, 91¹1'33”W). 
Table DR1. U-Pb and Hf isotope data

\begin{tabular}{|c|c|c|c|c|c|c|c|c|c|c|c|c|c|}
\hline \multirow{3}{*}{$\begin{array}{l}\text { MP1 } \\
\text { zrn001 }\end{array}$} & \multirow{2}{*}{\multicolumn{2}{|c|}{${ }^{207} \mathrm{~Pb} /{ }^{206} \mathrm{~Pb}(2 \sigma)$}} & \multirow[t]{2}{*}{${ }^{206} \mathrm{~Pb} /{ }^{238} \mathrm{U}(2 \sigma)$} & \multirow{2}{*}{\multicolumn{2}{|c|}{$\begin{array}{l}\text { U-Pb age }(2 \sigma) \S \\
\text { (Ma) }\end{array}$}} & \multicolumn{4}{|c|}{$\lambda^{176} \mathrm{Lu}: 1.865 \times 10^{-11}\left(\mathrm{yr}^{-1}\right)^{* * *}$} & \multicolumn{4}{|c|}{$\lambda^{176} \mathrm{Lu}: 1.983 \times 10^{-11}\left(\mathrm{yr}^{-1}\right)^{* *}$} \\
\hline & & & & & & \multicolumn{4}{|c|}{ nitial ${ }^{176} \mathrm{Hf}^{177} \mathrm{Hf}\left(2 \mathrm{~S} . \mathrm{E}_{\mathrm{Hf}(T) \text { DM DM1 }}(\mathrm{Ma})_{\text {DM2 }}(\mathrm{Ma})\right.$} & \multicolumn{4}{|c|}{ nitial ${ }^{176} \mathrm{Hf}^{177}{ }^{17 f}\left(2 \mathrm{S.E} \mathrm{Ef}_{\mathrm{H} T) \mathrm{DM} \text { DM1 }}(\mathrm{Ma})_{\mathrm{DM} 2}(\mathrm{Ma})\right.$} \\
\hline & 9 & \pm 0.003 & 9 & 1173 & \pm 73 & $5 \pm$ & -32 & 1323 & 1407 & $260 \pm 0.00$ & & 1276 & 1333 \\
\hline n002 & 74 & \pm 0.003 & 8 & 031 & \pm 88 & $4+$ & & & & $33 \pm 0$ & & & 025 \\
\hline 3 & 163 & \pm 0.034 & $7+0$ & 20 & \pm & $70 \pm 0$ & & & & $170 \pm 0$ & & & 926 \\
\hline 4 & 017 & \pm 0.006 & 19 & 24 & \pm & $07+00$ & & & & $07 \pm 0$. & & & 326 \\
\hline 5 & 0.086 & \pm 0.003 & $2998 \pm 0.00526$ & 1342 & \pm 72 & $58 \pm 0.000044$ & & & & $56 \pm 0.0$ & & & 676 \\
\hline 6 & $-\dagger$ & - & $5 \pm 0$ & 61 & \pm & $27 \pm 0.000059$ & -3 & & & $127 \pm 0.0$ & & & 2737 \\
\hline 7: & 0.073 & \pm 0.018 & $0.00211 \pm 0.00014$ & 14 & \pm & $2 \pm 0.000092$ & -42 & 2 & & $012 \pm 0.0$ & & 50 & 3064 \\
\hline : & 049 & \pm 0.009 & $0456 \pm 0.0$ & 29 & \pm & $3 \pm 0.00$ & & & & $83 \pm 0.0$ & -1 & & 381 \\
\hline 9 & ( & \pm 0.003 & $44652 \pm 0$ & 2544 & \pm 34 & $2 \pm$ & & & & $80 \pm 0$. & & & 3606 \\
\hline n010 & 093 & \pm 0.003 & $0 \pm 0$ & 1494 & \pm 61 & $7 \pm 0$. & & & & $06 \pm 0$. & & & 1904 \\
\hline n011 & 33 & \pm 0.005 & $37 \pm 0$ & 3073 & $\pm \quad 32$ & $5 \pm$ & & & & $12 \pm 0$. & & & 3073 \\
\hline n012: & 57 & \pm 0.005 & $0982 \pm 0$ & 63 & \pm & $3 \pm 0$ & -2 & 1 & & $163 \pm 0$ & & & 1903 \\
\hline n013 & 00 & \pm 0.001 & $8204 \pm 0.0$ & 1617 & $\pm \quad 24$ & $1 \pm 0.0$ & -15 & & & $70 \pm 0$ & & & 2598 \\
\hline n014 & 53 & \pm 0. & $1084 \pm 0.0$ & 2376 & \pm 64 & $0 \pm 0.0$ & & & & $26 \pm 0$. & & & 2481 \\
\hline 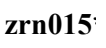 & 40 & \pm 0.012 & $6412 \pm 0.0$ & 980 & $\pm \quad 29$ & $4 \pm 0$ & & & & $04 \pm 0$. & & & 1657 \\
\hline n016 & 88 & \pm 0.002 & $7 \pm 0$ & 1383 & $\pm \quad 48$ & $25 \pm$ & & & & $22 \pm 0$. & & & 1721 \\
\hline n017 & 096 & \pm 0.007 & $7090 \pm 0.0$ & 1545 & \pm 145 & $2 \pm 0$ & -1 & & & $01 \pm 0$ & & & 2348 \\
\hline 8 & 945 & \pm 0.003 & $5 \pm 0$ & 243 & \pm & $2 \pm 0$ & -22 & & & $12 \pm 0$ & & & 1853 \\
\hline 19 & 74 & \pm 0.002 & $7 \pm 0$ & 1032 & \pm 62 & $3 \pm 0$ & & & & $62 \pm 0$ & & & 1463 \\
\hline n020 & 113 & \pm 0.007 & $6386 \pm 0$ & 1841 & \pm 114 & $35 \pm 0$ & & & & $84 \pm 0$. & & & 1875 \\
\hline rn021 & 0.085 & \pm 0.003 & $24557 \pm 0$ & 1321 & \pm 70 & $38 \pm 0$. & & & 18 & $37 \pm 0$ & -6 & & 1744 \\
\hline rn022 & 0.091 & \pm 0.002 & $5 \pm 0$. & 1451 & $\pm \quad 46$ & $1 \pm 0$ & & 1 & 19 & $950 \pm 0$. & -5 & & 1834 \\
\hline rn023 & 104 & \pm 0.003 & $29437 \pm 0$ & 1701 & \pm 51 & $6 \pm 0$ & & 1 & 17 & $32 \pm 0$ & & & 1701 \\
\hline 1024 & 54 & \pm 0.011 & $6456 \pm 0$ & 2388 & \pm 125 & $1 \pm 0$ & -15 & 3 & 32 & $997 \pm 0$. & -13 & ? & 3246 \\
\hline rn025 & 075 & \pm 0.011 & $0435 \pm 0.00020$ & 1184 & $\pm \quad 42$ & $88 \pm 0$. & & 16 & 18 & $086 \pm 0$ & -8 & 8 & 1752 \\
\hline rn026 & 079 & \pm 0.002 & $21777 \pm 0.00713$ & 966 & \pm 91 & $07 \pm 0.000037$ & -10 & 14 & 17 & $206 \pm 0.0$ & -9.9 & 1412 & 1663 \\
\hline zrn027 & 971 & \pm 0.003 & $22341 \pm 0.00772$ & 1150 & $\pm \quad 44$ & $167 \pm 0.000$ & & 14 & 16 & $165 \pm 0.0$ & -6 & 3 & 1590 \\
\hline n028 & 0.078 & \pm 0.002 & $17492 \pm 0.00573$ & 1482 & \pm 102 & $81877 \pm 0.000$ & & & 20 & $374 \pm 0$. & & & 1986 \\
\hline & 0.093 & \pm 0.005 & $0.06031 \pm 0.00183$ & 35 & \pm & $0.281802 \pm 0.000047$ & $-4 c$ & & & $802 \pm 0.0$ & $-4 c$ & & 3555 \\
\hline zrn030 & - & - & $0.00552 \pm 0.00077$ & 896 & \pm 27 & $0.282096 \pm 0.000055$ & -16 & & & $282094 \pm 0.0$ & -1 & & 2007 \\
\hline zrn031 & 0.042 & \pm 0.011 & $0.14920 \pm 0.00490$ & 28 & \pm & $82155 \pm 0.000042$ & -37 & 17 & & $0.282154 \pm 0.00$ & -37.4 & 1725 & 2703 \\
\hline rn032 & 0.105 & \pm 0.002 & $0.32477 \pm 0.01061$ & 1717 & $\pm \quad 32$ & $281716 \pm 0.000053$ & & 2085 & 22 & $0.281712 \pm 0.000053$ & -6.5 & 2004 & 2164 \\
\hline rn033 & 0.083 & \pm 0.001 & $0.21132 \pm 0.00684$ & 1274 & $\pm \quad 29$ & $281985 \pm 0.000054$ & -10.5 & 1754 & 2017 & $0.281980 \pm 0.000054$ & -9.4 & 1693 & 1926 \\
\hline
\end{tabular}


$\begin{array}{c:cccc}\text { zrn034 } & 0.041 & \pm 0.005 & 0.00633 \pm 0.00025 & 41 \pm 2\end{array}$

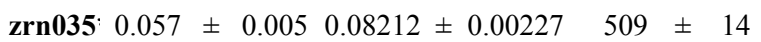

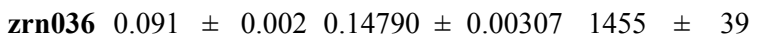
$\begin{array}{lllllll}\text { zrn037 } & 0.109 & \pm & 0.003 & 0.29436 & \pm 0.00737 \quad 1778 & \pm 45\end{array}$

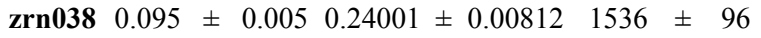
zrn039 $0.101 \pm 0.0020 .28015 \pm 0.00840 \quad 1649 \pm 35$ $\begin{array}{llllll}\text { zrn040 } & 0.046 \pm 0.006 & 0.01029 \pm 0.00043 \quad 66 \pm 3\end{array}$

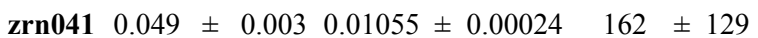
zrn042 $0.176 \pm 0.0020 .51215 \pm 0.010452618 \pm 22$ $\begin{array}{lllllll}\text { zrn043 } & 0.059 & \pm 0.006 & 0.02513 & \pm 0.00078 & 160 \pm 5\end{array}$ $\begin{array}{llllll}\text { zrn044 } & 0.059 & \pm 0.013 & 0.00143 \pm 0.00008 & 9 & \pm\end{array}$

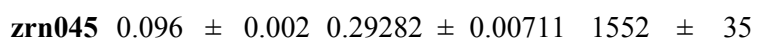

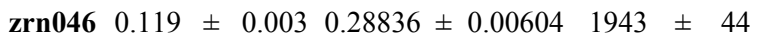

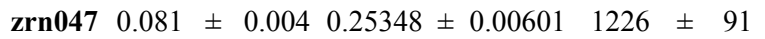
$\begin{array}{llllll}\operatorname{zrn048} & 0.038 & \pm 0.009 & 0.01078 \pm 0.00052 & 69 & \pm\end{array}$ zrn049: $0.061 \pm 0.0050 .02506 \pm 0.00071 \quad 160 \pm 4$

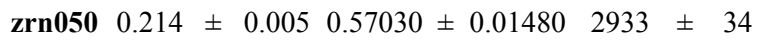
$\begin{array}{lllllll}\text { zrn051 } & 0.087 & \pm & 0.014 & 0.00886 \pm 0.00028 & 57 & \pm\end{array}$

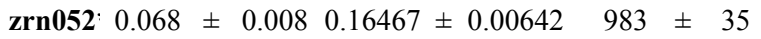

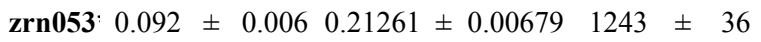

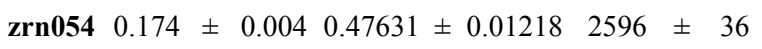

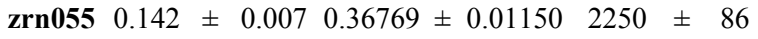
zrn056 $0.073 \pm 0.0020 .19056 \pm 0.00404 \quad 1015 \pm 68$

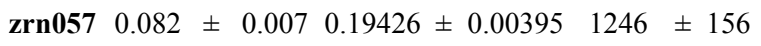
zrn058 $0.076 \pm 0.003 \quad 0.18553 \pm 0.00330 \quad 1096 \pm 67$ $\begin{array}{lllllll}\text { zrn059: } & 0.048 & \pm 0.004 & 0.02783 & \pm 0.00070 & 177 & \pm 4\end{array}$ $\begin{array}{lllllll}\operatorname{zrn060} & 0.069 & \pm 0.012 & 0.00313 & \pm 0.00015 & 20 & \pm\end{array}$

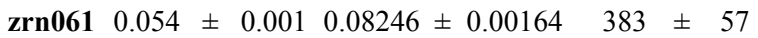

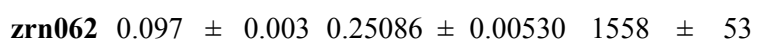
zrn063 $0.250 \pm 0.0040 .53313 \pm 0.00883 \quad 3186 \pm 26$

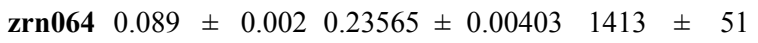
$\begin{array}{lllllll}\operatorname{zrn065} & 0.075 & \pm & 0.005 & 0.18474 \pm 0.00459 & 1093 & \pm 25\end{array}$ zrn066 $0.105 \pm 0.003 \quad 0.26891 \pm 0.00729 \quad 1708 \quad \pm 51$ $\begin{array}{lllllll}\operatorname{zrn067} & 0.096 & \pm 0.002 & 0.27524 & \pm 0.00717 & 1554 & \pm 35\end{array}$ $\begin{array}{llllll}\text { zrn068} & 0.023 & \pm 0.014 & 0.00994 \pm 0.00036 & 64 & \pm 2\end{array}$

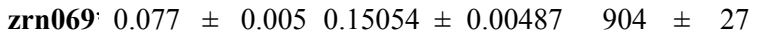
zrn070 $0.174 \pm 0.003 \quad 0.49304 \pm 0.00720 \quad 2593 \pm 25$
$0.282183 \pm 0.000036-35.9$ $0.282598 \pm 0.000054-9.0$ $0.281898 \pm 0.000036 \quad-8.7$ $0.281875 \pm 0.000047 \quad-0.8$ $0.282057 \pm 0.000033 \quad-0.9$ $0.281915 \pm 0.000054 \quad-2.9$ $0.282618 \pm 0.000034-19.9$ $0.282709 \pm 0.000052-14.2$ $0.280927 \pm 0.000049-11.6$ $0.282903 \pm 0.000046 \quad-7.4$ $0.282615 \pm 0.000041-21.5$ $0.281927 \pm 0.000050 \quad-5.1$ $0.281553 \pm 0.000033-7.8$ $0.282075 \pm 0.000039 \quad-8.6$ $0.282762 \pm 0.000041-14.7$ $0.282919 \pm 0.000040-6.8$ $0.281039 \pm 0.000068 \quad 1.0$ $0.282934 \pm 0.000052-9.0$ $0.282298 \pm 0.000056-7.1$ $0.281990 \pm 0.000044-11.1$ $0.281042 \pm 0.000048 \quad-8.1$ $0.281277 \pm 0.000038$ $0.282299 \pm 0.000049$ $0.282106 \pm 0.000051$ $0.282258 \pm 0.000049-5.5$ $0.282638 \pm 0.000037-16.3$ $0.283038 \pm 0.000042-6.3$ $0.282567 \pm 0.000042-13.4$ $0.282113 \pm 0.000043 \quad 1.7$ $0.280677 \pm 0.000046 \quad-4.8$ $0.281952 \pm 0.000056 \quad-7.9$ $0.282221 \pm 0.000033 \quad-6.9$ $0.281826 \pm 0.000073 \quad-4.5$ $0.281951 \pm 0.000040 \quad-4.2$ $0.282788 \pm 0.000051-14.0$ $0.282123 \pm 0.000036-15.4$ $0.281152 \pm 0.000047 \quad-4.3$

Iizuka et al., Table DR1 p.2

932

1853

1815

1577

1737

1008

834

3134

510

1026

1783

2295

1619

768

483

2933

481

1312

1753

2958

2666

1303

1564

1351

948

318

1016

1558

3399

1775

1413

1910

1745

727

1620

2786
2070

1838

1601

1737

1534

1210

3402

710

1591

1911

2484

1835

1160

669

2933

725

1496

2032

3146

2884

1464

1740

1494

1378

487

1366

1558

3507

1972

1590

2022

1850

1099

2006

2887
$0.282183 \pm 0.000036-36.0$ $0.282597 \pm 0.000054-8.6$

$0.281891 \pm 0.000036-7.5$

$0.281873 \pm 0.000047$

$0.282055 \pm 0.000033$

$0.281911 \pm 0.000054$

$0.282618+0.000034-19.9$

$0.282708 \pm 0.000052-14.1$

$0.280924 \pm 0.000049-9.0$

$0.282902 \pm 0.000046-7.3$

$0.282615 \pm 0.000041-21.6$

$0.281926 \pm 0.000050-3.6$

$0.281549 \pm 0.000033-5.9$

$0.282073 \pm 0.000039 \quad-7.5$

$0.282762 \pm 0.000041-14.8$

$0.282918 \pm 0.000040-6.8$

$0.281036 \pm 0.000069$

$0.282934 \pm 0.000052-9.0$

$0.282298 \pm 0.000056-6.2$

$0.281988 \pm 0.000044-10.0$

$0.281036 \pm 0.000047 \quad-5.6$

$0.281274 \pm 0.000038-7.0$

$0.282297 \pm 0.000049 \quad-5.4$

$0.282104 \pm 0.000051-5.8$

$0.282255 \pm 0.000049-4.6$

$0.282638 \pm 0.000037-16.2$

$0.283038 \pm 0.000042-6.4$

$0.282565 \pm 0.000042-13.2$

$0.282098 \pm 0.000046 \quad 2.7$

$0.280671+0.000046-1.6$

$0.281950 \pm 0.000056-6.6$

$0.282221 \pm 0.000033-5.9$

$0.281825 \pm 0.000073-2.8$

$0.281949 \pm 0.000040 \quad-2.7$

$0.282787 \pm 0.000051-14.0$

$0.282122 \pm 0.000036-14.6$

$0.281151 \pm 0.000047-1.6$
$675 \quad 2618$

$901 \quad 1130$

$1789 \quad 1973$

$1778 \quad 1778$

$1711 \quad 1743$

$977 \quad 1510$

$808 \quad 1188$

$3002 \quad 3208$

$497 \quad 697$

$995 \quad 1572$

$1712 \quad 1798$

$2202 \quad 2346$

$1559 \quad 1744$

$746 \quad 1144$

$471 \quad 657$

$2933 \quad 2933$

$471 \quad 717$

$1261 \quad 1422$

$1688 \quad 1935$

$2837 \quad 2966$

$2554 \quad 2718$

$1256 \quad 1391$

$1505 \quad 1649$

$1303 \quad 1420$

$918 \quad 1352$

$313 \quad 488$

$983 \quad 1333$

$1558 \quad 1558$

$3253 \quad 3289$

$1707 \quad 1869$

$1358 \quad 1508$

$1832 \quad 1898$

$1674 \quad 1738$

$706 \quad 1084$

$1560 \quad 1929$

$2664 \quad 2702$ 
Iizuka et al., Table DR1 p.3

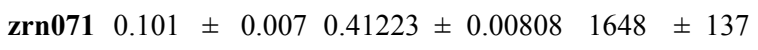

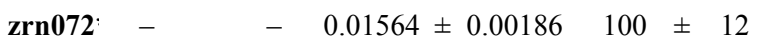
$\begin{array}{lllllll}\text { zrn073 } & 0.086 & \pm & 0.002 & 0.21946 \pm 0.00341 & 1333 & \pm\end{array}$

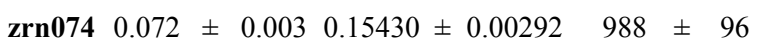
zrn075 $0.086 \pm 0.0020 .23836 \pm 0.00381 \quad 1335 \pm 53$ $\begin{array}{llllll}\text { zrn076 } & 0.051 & \pm 0.003 & 0.04212 \pm 0.00159 & 238 & \pm 144\end{array}$ zrn077 $0.073 \pm 0.0020 .15385 \pm 0.00553 \quad 1016 \pm 58$ $\begin{array}{llllllll}\operatorname{zrn078} & 0.185 & \pm & 0.003 & 0.50817 & \pm 0.01804 & 2697 & \pm 24\end{array}$ $\begin{array}{llllll}\text { zrn079 } & 0.103 & \pm & 0.002 & 0.30365 \pm 0.01076 \quad 1672 & \pm 32\end{array}$

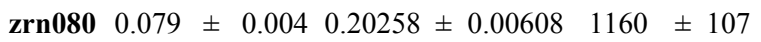

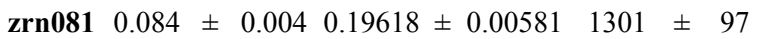
$\begin{array}{lllllll}\text { zrn082 } & 0.099 & \pm & 0.003 & 0.24421 & \pm 0.00673 \quad 1613 & \pm 60\end{array}$ $\begin{array}{lllllll}\operatorname{zrn083} & 0.084 & \pm & 0.003 & 0.21990 \pm 0.00803 & 1291 & \pm 66\end{array}$

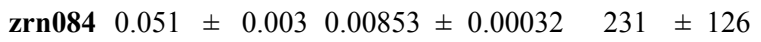
$\begin{array}{lllllll}\operatorname{zrn085} & 0.059 & \pm & 0.005 & 0.02069 \pm 0.00085 & 132 & \pm\end{array}$ $\begin{array}{lllllll}\text { zrn086 } & 0.045 & \pm & 0.003 & 0.02722 \pm 0.00079 & 173 & \pm\end{array}$

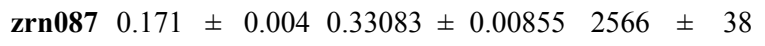
$\begin{array}{lllllll}\text { zrn088} & 0.040 & \pm & 0.014 & 0.00293 & \pm 0.00021 & 19 \pm 1\end{array}$ $\begin{array}{lllllll}\text { zrn089 } & 0.052 & \pm 0.004 & 0.02457 & \pm 0.00075 & 156 & \pm\end{array}$ $\begin{array}{lllllll}\operatorname{zrn090} & 0.051 & \pm 0.005 & 0.02974 \pm 0.00123 & 189 & \pm & 8\end{array}$

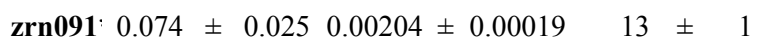
zrn092 $0.089 \pm 0.004 \quad 0.21943 \pm 0.00590 \quad 1406 \pm 77$ $\begin{array}{llllll}\text { zrn093 } & 0.046 \pm 0.004 & 0.00376 \pm 0.00012 \quad 24 \pm 1\end{array}$ $\begin{array}{llllll}\text { zrn094: } & 0.080 & \pm 0.017 & 0.01090 \pm 0.00070 & 70 \pm 4\end{array}$

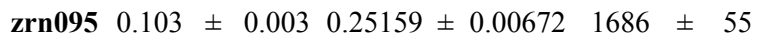
zrn096 $0.202 \pm 0.0040 .48912 \pm 0.01292 \quad 2845 \pm 30$

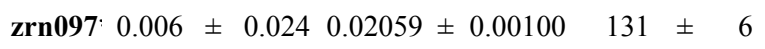
$\begin{array}{llllll}\text { zrn098 } & 0.081 & \pm 0.003 & 0.14184 \pm 0.00445 \quad 1214 & \pm 68\end{array}$ $\begin{array}{llllll}\text { zrn099 } & 0.103 & \pm 0.002 & 0.26111 \pm 0.00810 & 1671 & \pm 35\end{array}$

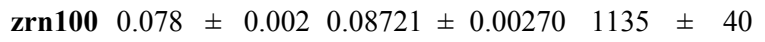

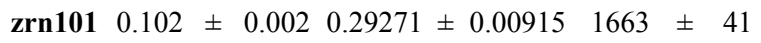

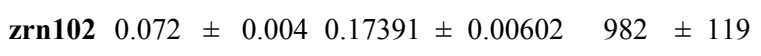
$\begin{array}{lllllll}\text { zrn103 } & 0.089 & \pm 0.011 & 0.01064 \pm 0.00048 & 68 & \pm\end{array}$ zrn104: $0.060 \pm 0.007 \quad 0.09811 \pm 0.00375 \quad 603 \pm 22$

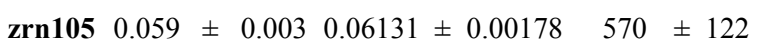

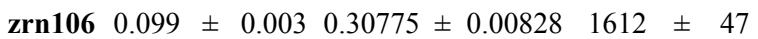
$\operatorname{zrn107} 0.107 \pm 0.0020 .30184 \pm 0.00791 \quad 1755 \pm 33$
$0.281451 \pm 0.000037-19.4$ $0.282784 \pm 0.000039-13.1$ $0.282051 \pm 0.000056 \quad-6.6$ $0.282317 \pm 0.000048 \quad-6.3$ $0.281848 \pm 0.000032-13.7$ $0.282625 \pm 0.000033-15.2$ $0.282375 \pm 0.000052-3.5$ $0.281126 \pm 0.000032 \quad-2.4$ $0.281718 \pm 0.000028 \quad-9.2$ $0.282211 \pm 0.000058 \quad-5.5$ $0.282290 \pm 0.000025 \quad 1.1$ $0.281804 \pm 0.000035-7.8$ $0.282181 \pm 0.000037-3.0$ $0.282798 \pm 0.000057 \quad-9.2$ $0.282413 \pm 0.000045 \quad-25.4$ $0.282551 \pm 0.000030-19.5$ $0.281036 \pm 0.000032 \quad-9.2$ $0.282525 \pm 0.000047 \quad-24.4$ $0.282840 \pm 0.000041 \quad-9.7$ $0.282501 \pm 0.000030 \quad-20.8$ $0.282629 \pm 0.000021 \quad-20.9$ $0.282148 \pm 0.000039-1.1$ $0.282987 \pm 0.000021 \quad-8.0$ $0.282396 \pm 0.000037 \quad-27.6$ $0.281930 \pm 0.000047 \quad-1.4$ $0.280789 \pm 0.000055-10.3$ $0.282373 \pm 0.000024 \quad-26.9$ $0.282067 \pm 0.000070 \quad-9.2$ $0.281748 \pm 0.000028 \quad-8.2$ $0.282064 \pm 0.000032-11.4$ $0.281820 \pm 0.000027 \quad-5.9$ $0.282266 \pm 0.000042 \quad-8.3$ $0.282281 \pm 0.000052-31.7$ $0.282018 \pm 0.000023-27.1$ $0.282276 \pm 0.000032-18.8$ $0.281811 \pm 0.000028 \quad-7.6$ $0.281579 \pm 0.000029-12.0$ 721 1633 1281 1961 955 1179 2804 2092 1414 1301 1968 1431
1074

1799

1444

1354

1271

2859

2319

1555

1301

2159

1510

913

1993

1603

3187

1813

876

1716

1552

1489

615

2095

1781

3532

2096

1866

2247

1946

2077

1578

2388

2548

1930

2142

2587
$0.281449 \pm 0.000037-17.8$ $0.282784 \pm 0.000039-13.2$

$0.282048 \pm 0.000056-5.4$

$0.282316 \pm 0.000048 \quad-5.4$

$0.281847 \pm 0.000032-12.4$

$0.282625 \pm 0.000033-15.0$

$0.282374 \pm 0.000052-2.6$

$0.281125 \pm 0.000032$

$0.281717 \pm 0.000028-7.6$

$0.282209 \pm 0.000058-4.5$

$0.282289 \pm 0.000025 \quad 2.3$

$0.281802 \pm 0.000035-6.3$

$0.282181 \pm 0.000037-1.8$

$0.282798 \pm 0.000057-9.1$

$0.282413 \pm 0.000045-25.4$

$0.282551 \pm 0.000030-19.4$

$0.281033 \pm 0.000032-6.6$

$0.282525 \pm 0.000047 \quad-24.5$

$0.282840 \pm 0.000041-9.7$

$0.282501 \pm 0.000030-20.7$

$0.282629 \pm 0.000021-21.0$

$0.282147 \pm 0.000039 \quad 0.2$

$0.282987 \pm 0.000021 \quad-8.0$

$0.282396 \pm 0.000037-27.7$

$0.281928 \pm 0.000047 \quad 0.3$

$0.280783 \pm 0.000055-7.5$

$0.282373 \pm 0.000024-26.8$

$0.282056 \pm 0.000070 \quad-8.4$

$0.281745 \pm 0.000028-6.6$

$0.282060+0.000032-10.4$

$0.281818 \pm 0.000027 \quad-4.3$

$0.282265 \pm 0.000042-7.4$

$0.282281 \pm 0.000052-31.8$

$0.282018 \pm 0.000023-26.6$

$0.282276 \pm 0.000032-18.3$

$0.281810 \pm 0.000028-6.0$

$0.281577 \pm 0.000029-10.3$
$2427 \quad 2857$

$702 \quad 1057$

$1572 \quad 1704$

$1233 \quad 1372$

$1887 \quad 2192$

$924 \quad 1325$

$1134 \quad 1201$

$2697 \quad 2697$

$2008 \quad 2190$

$1361 \quad 1474$

$1301 \quad 1301$

$1890 \quad 2040$

$1372 \quad 1417$

$647 \quad 892$

$1289 \quad 1957$

$1057 \quad 1574$

$2850 \quad 3002$

$1139 \quad 1790$

$598 \quad 860$

$1135 \quad 1683$

$972 \quad 1534$

$1406 \quad 1406$

$394 \quad 614$

$1331 \quad 2059$

$3163 \quad 3332$

$1353 \quad 2057$

$1589 \quad 1799$

$1965 \quad 2123$

$1601 \quad 1861$

$1853 \quad 1957$

$1315 \quad 1504$

$1514 \quad 2349$

$1797 \quad 2475$

$1398 \quad 1871$

$1878 \quad 2023$

$2205 \quad 2452$ 
$\begin{array}{cccccc}\text { zrn108 } & 0.056 & \pm 0.009 & 0.00975 & \pm 0.00043 \quad 63 & \pm 3\end{array}$ zrn109: - $\quad-\quad 0.00160 \pm 0.00273 \quad 10 \pm 18$ $\begin{array}{llllll}\text { zrn110 } & 0.104 & \pm 0.004 & 0.26906 \pm 0.00640 & 1704 & \pm 68\end{array}$

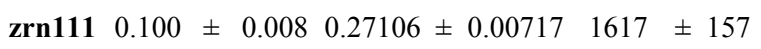
$\begin{array}{llllll}\text { zrn112 } & 0.120 & \pm 0.243 & 0.00243 & \pm 0.00075 \quad 16 & \pm\end{array}$ zrn113 $0.197 \pm 0.0040 .20473 \pm 0.004762798 \pm 30$ zrn114: $0.043 \pm 0.0050 .01388 \pm 0.00047 \quad 89 \pm 3$ zrn115 $0.092 \pm 0.0030 .10275 \pm 0.00304 \quad 1468 \pm 57$ zrn116 - $\quad-\quad 0.00930 \pm 0.00082 \quad 60 \pm 5$

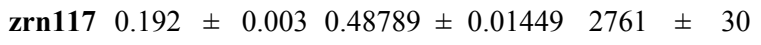
zrn118 $0.091 \pm 0.003 \quad 0.21918 \pm 0.00662 \quad 1450 \pm 57$

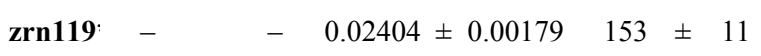
$\begin{array}{lllllll}\operatorname{zrn120} & 0.077 & \pm & 0.005 & 0.17100 \pm 0.00479 & 1110 & \pm 121\end{array}$ $\begin{array}{llllll}\text { zrn121 } & 0.024 & \pm 0.059 & 0.00358 \pm 0.00029 & 23 & \pm 2\end{array}$ $\begin{array}{llllll}\text { zrn122 } & 0.052 \pm 0.005 & 0.06702 \pm 0.00206 & 418 & \pm 12\end{array}$ $\begin{array}{lllll}\text { zrn123 } & 0.058 & \pm 0.0220 .01356 \pm 0.00055 & 87 & \pm 4\end{array}$ $\begin{array}{lllllll}\text { zrn124 } & 0.181 & \pm & 0.004 & 0.51802 \pm 0.01261 & 2659 & \pm\end{array}$

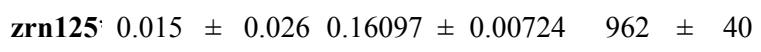
zrn126 $0.156 \pm 0.0040 .17079 \pm 0.00393 \quad 2414 \pm 40$

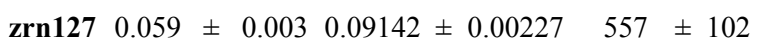

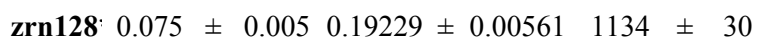

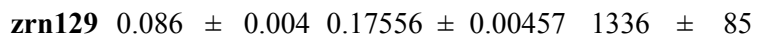
$\begin{array}{lllllll}\text { zrn130 } & 0.004 & \pm 0.044 & 0.00402 \pm 0.00034 & 26 & \pm 2\end{array}$ $\begin{array}{llllll}\text { zrn131 } & 0.088 & \pm 0.004 & 0.23275 & \pm 0.00626 \quad 1372 & \pm 92\end{array}$ zrn132 $0.089 \pm 0.0060 .25194 \pm 0.00610 \quad 1413 \pm 133$ $\begin{array}{lllllll}\text { zrn133 } & 0.103 & \pm & 0.004 & 0.23405 & \pm 0.00576 \quad 1670 & \pm\end{array}$

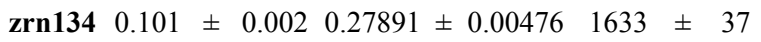

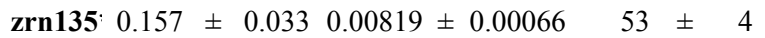

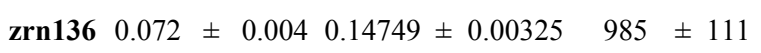

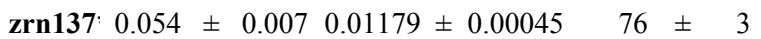
$\begin{array}{llllllll}\text { zrn138 } & 0.227 & \pm & 0.005 & 0.56486 & \pm 0.01412 & 3034 & \pm\end{array}$ $\begin{array}{lllllll}\text { zrn139 } & 0.100 & \pm & 0.007 & 0.09145 \pm 0.00233 & 1628 & \pm 134\end{array}$ zrn140 - $\quad-0.00873 \pm 0.00128 \quad 56 \pm 8$

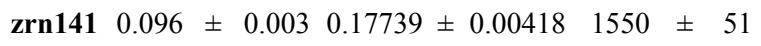

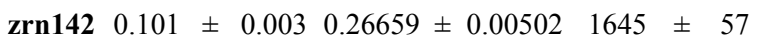

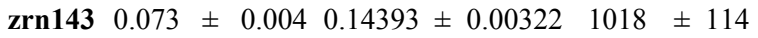
$\operatorname{zrn144} 0.110 \pm 0.007 \quad 0.28454 \pm 0.00811 \quad 1614 \pm 41$
$0.282741 \pm 0.000026-15.6$ $0.283081 \pm 0.000041-5.0$ $0.281859 \pm 0.000044 \quad-3.4$ $0.281993 \pm 0.000036-1.0$ $0.282554 \pm 0.000045-23.5$ $0.280914 \pm 0.000037 \quad-7.1$ $0.282146 \pm 0.000047 \quad-36.0$ $0.281693 \pm 0.000033-15.6$ $0.282080 \pm 0.000030-39.1$ $0.281133 \pm 0.000041 \quad-0.4$ $0.282055 \pm 0.000033-3.3$ $0.282525 \pm 0.000034-20.9$ $0.282070 \pm 0.000033-11.8$ $0.283009 \pm 0.000026 \quad-7.2$ $0.282202 \pm 0.000027 \quad-25.4$ $0.282314 \pm 0.000028-30.1$ $0.280880 \pm 0.000024-12.2$ $0.282218 \pm 0.000037-10.5$ $0.280969 \pm 0.000058-15.7$ $0.282001 \pm 0.000029 \quad-28.9$ $0.282148 \pm 0.000031 \quad-8.4$ $0.282203 \pm 0.000037-1.1$ $0.282553 \pm 0.000030-23.2$ $0.282104 \pm 0.000043 \quad-3.6$ $0.281894 \pm 0.000041-10.0$ $0.281863 \pm 0.000064 \quad-4.1$ $0.281931 \pm 0.000037 \quad-2.7$ $0.281892 \pm 0.000036-45.9$ $0.282216 \pm 0.000043-10.0$ $0.281800 \pm 0.000040 \quad-48.5$ $0.280967 \pm 0.000036 \quad 1.3$ $0.281637 \pm 0.000051-13.3$ $0.282008 \pm 0.000059-41.7$ $0.281779 \pm 0.000026-10.4$ $0.281769 \pm 0.000022 \quad-8.2$ $0.282206 \pm 0.000043 \quad-9.4$ $0.281843 \pm 0.000028 \quad-6.4$

Iizuka et al., Table DR1 p.4

247

1858

1662

1126

3115

1776

2183

1892

2779

1600

1140

1654

366

1606

1502

3199

1448

3113

1900

1522

1385

1125

1539

1868

1859

1758

2197

1446

2340

3034

2233

2008

2022

2017

1454

1905

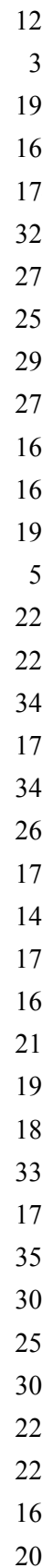

1219

$0.282741 \pm 0.000026-15.7$

$0.283081 \pm 0.000041-5.1$

$0.281858 \pm 0.000044-1.7$

$0.281991 \pm 0.000036 \quad 0.6$

$0.282554 \pm 0.000045-23.6$

$0.280908 \pm 0.000037-4.3$

$0.282146 \pm 0.000047-36.0$

$0.281689 \pm 0.000033-14.3$

$0.282080 \pm 0.000030-39.1$

$0.281128 \pm 0.000041 \quad 2.4$

$0.282052 \pm 0.000033 \quad-2.0$

$0.282525 \pm 0.000034-20.9$

$0.282069 \pm 0.000033-10.8$

$561 \quad 0.283008 \pm 0.000026-7.3$

$2259 \quad 0.282202 \pm 0.000027-25.1$

$2887 \quad 0.282314 \pm 0.000028-30.1$

$478 \quad 0.280877 \pm 0.000024-9.5$

$\begin{array}{lll}716 & 0.282217 \pm 0.000037 & -9.7\end{array}$

$474 \quad 0.280951 \pm 0.000058-13.9$

$2632 \quad 0.282001 \pm 0.000029-28.4$

$1736 \quad 0.282147 \pm 0.000031-7.4$

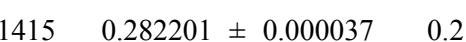

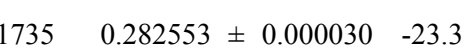

$\begin{array}{lll}1632 & 0.282103 \pm 0.000043 \quad-2.3\end{array}$

$2116 \quad 0.281887 \pm 0.000041 \quad-8.8$

$1963 \quad 0.281854 \pm 0.000064 \quad-2.8$

$\begin{array}{lll}1825 & 0.281928 \pm 0.000037 & -1.2\end{array}$

$3372 \quad 0.281892 \pm 0.000036-45.9$

$\begin{array}{lll}1701 & 0.282215 \pm 0.000043 \quad-9.1\end{array}$

$3581 \quad 0.281800 \pm 0.000040-48.6$

$3034 \quad 0.280965 \pm 0.000036 \quad 4.5$

$2559 \quad 0.281632 \pm 0.000051-11.9$

$3083 \quad 0.282008 \pm 0.000059-41.7$

$2278 \quad 0.281778 \pm 0.000026 \quad-8.9$

$2216 \quad 0.281768 \pm 0.000022-6.6$

$16950.282205 \pm 0.000043 \quad-8.5$

$2062 \quad 0.281841 \pm 0.000028 \quad-4.8$
$780 \quad 1202$

$245 \quad 386$

$1781 \quad 1824$

$1617 \quad 1617$

$1092 \quad 1723$

$2984 \quad 3081$

$1721 \quad 2662$

$2100 \quad 2449$

$1833 \quad 2854$

$2761 \quad 2761$

$1104 \quad 1658$

$1592 \quad 1865$

$359 \quad 560$

$1553 \quad 2199$

$1457 \quad 2246$

$3064 \quad 3280$

$1396 \quad 1642$

$3010 \quad 3328$

$1835 \quad 2561$

$1465 \quad 1651$

$1336 \quad 1336$

$1091 \quad 1714$

$1477 \quad 1534$

$1805 \quad 2023$

$1795 \quad 1864$

$1689 \quad 1719$

$2129 \quad 3318$

$1393 \quad 1623$

$2266 \quad 3518$

$3034 \quad 3034$

$2150 \quad 2438$

$1946 \quad 3032$

$1942 \quad 2159$

19352096

$1401 \quad 1617$

$1829 \quad 1948$ 
Iizuka et al., Table DR1 p.5

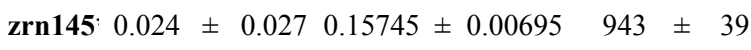
zrn146 $0.083 \pm 0.004 \quad 0.26069 \pm 0.00550 \quad 1270 \pm 84$ zrn147 $0.062 \pm 0.0140 .22197 \pm 0.00753 \quad 1292 \pm 40$

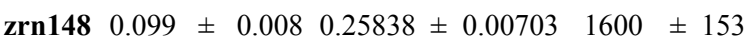

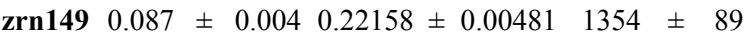
$\begin{array}{lllllll}\text { zrn150 } & 0.084 & \pm 0.004 & 0.19882 & \pm 0.00451 & 1301 & \pm 100\end{array}$ zrn151 - $\quad-\quad-0.00055 \pm 0.00109 \quad 4 \pm 7$

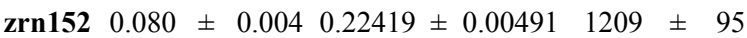

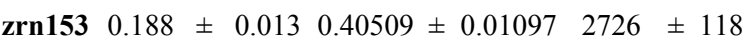

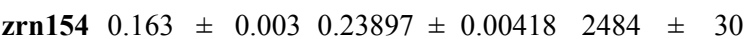

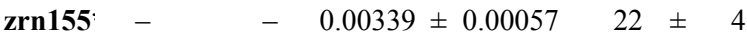
$\begin{array}{lllllll}\text { zrn156 } & 0.182 & \pm 0.004 & 0.53857 & \pm 0.01478 \quad 2671 & \pm 35\end{array}$ zrn157 $0.082 \pm 0.0040 .21792 \pm 0.006501246 \pm 95$

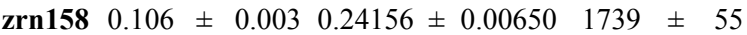

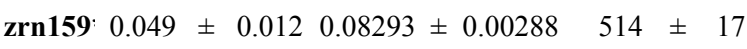

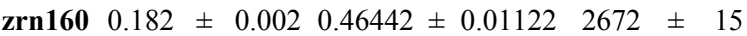
zrn161 $0.196 \pm 0.0050 .19454 \pm 0.004912797 \pm 41$

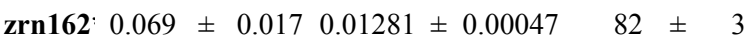

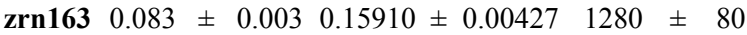
zrn164: $0.081 \pm 0.007 \quad 0.17052 \pm 0.00585 \quad 1015 \pm 32$ zrn165 $0.045 \pm 0.0060 .01548 \pm 0.00058 \quad 99 \pm 4$

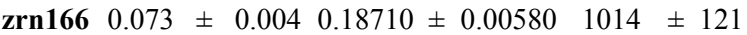
zrn167 $0.038 \pm 0.0050 .01111 \pm 0.00041 \quad 71 \pm 3$

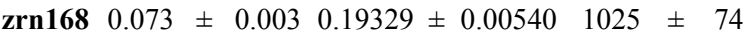

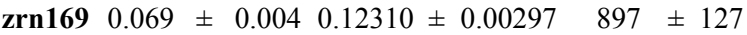

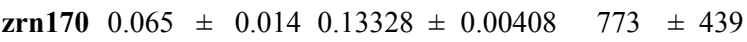

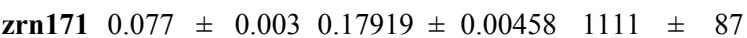

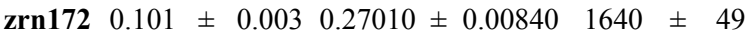
$\begin{array}{llllllll}\text { zrn173 } & 0.102 & \pm & 0.004 & 0.25354 & \pm 0.00788 & 1659 & \pm\end{array}$ zrn174 $0.085 \pm 0.0030 .22115 \pm 0.005461321 \pm 68$ zrn175 $0.085 \pm 0.0050 .22285 \pm 0.00630 \quad 1305 \pm 110$

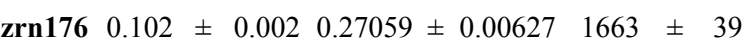
zrn177 $0.076 \pm 0.0050 .19351 \pm 0.00575 \quad 1140 \pm 31$

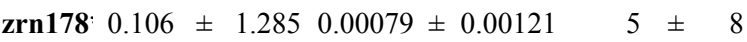

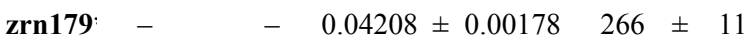
zrn180 $0.080 \pm 0.003 \quad 0.17657 \pm 0.00638 \quad 1189 \pm 79$ zrn181 $0.061 \pm 0.0050 .06386 \pm 0.00252 \quad 399 \pm 15$
$0.282234 \pm 0.000038-10.5$ $0.282007 \pm 0.000051-9.8$ $0.282085 \pm 0.000052 \quad-6.4$ $0.281875 \pm 0.000052 \quad-5.6$ $0.281896 \pm 0.000037-11.5$ $0.282095 \pm 0.000039-5.9$ $0.282390 \pm 0.000040-29.6$ $0.282151 \pm 0.000028-6.3$ $0.281160 \pm 0.000084-0.4$ $0.280892 \pm 0.000042-16.5$ $0.282625 \pm 0.000054-20.8$ $0.281052 \pm 0.000071 \quad-5.7$ $0.282230 \pm 0.000076 \quad-2.5$ $0.281864 \pm 0.000041 \quad-2.3$ $0.282020 \pm 0.000037-29.3$ $0.281086 \pm 0.000064 \quad-4.5$ $0.280771 \pm 0.000038-12.2$ $0.282494 \pm 0.000044 \quad-23.9$ $0.282206 \pm 0.000049 \quad-2.5$ $0.282144 \pm 0.000027-11.7$ $0.282502 \pm 0.000034-23.1$ $0.282083 \pm 0.000036-13.9$ $0.282358 \pm 0.000043-29.0$ $0.281921 \pm 0.000064-19.4$ $0.282419 \pm 0.000049 \quad-5.1$ $0.282126 \pm 0.000030-18.8$ $0.282083 \pm 0.000053-11.3$ $0.281828 \pm 0.000051 \quad-6.2$ $0.281576 \pm 0.000061-14.6$ $0.282088 \pm 0.000045 \quad-5.6$ $0.282164 \pm 0.000026-3.3$ $0.281718 \pm 0.000039-9.5$ $0.282115 \pm 0.000043 \quad-9.4$ $0.282693 \pm 0.000036-18.8$ $0.282613 \pm 0.000050-14.9$ $0.282115 \pm 0.000037-8.1$ $0.282442 \pm 0.000039-17.4$
1720

1587

1856

1879

1570

1397

1499

2744

3219

1006

2927

1363

1842

1879

2872

3338

1208

1394

1556

1191

1656

1435

1919

1136

1645

1632

1924

2323

1576

1457

2095

1575

898

967

1563

1218

\section{0}

1696

1967

1750

1997

2166

1719

2170

1660

2753

3600

1554

3059

1430

1898

2625

2976

3616

1835

1459

1854

1798

2009

2192

2402

1267

2120

1919

2076

2680

1718

1542

2329

1815

1396

1357

1770

1671
$0.282233 \pm 0.000038$

$0.282004+0.00005$

$0.282084 \pm 0.00005$

$0.281872 \pm 0.000052$

$0.281895 \pm 0.000037-10.2$

$0.282092 \pm 0.000039-4.7$

$0.282390 \pm 0.000040-29.7$

$0.282150 \pm 0.000028-5.2$

$0.281159 \pm 0.000084 \quad 2.5$

$0.280870 \pm 0.000042-14.7$

$0.282625 \pm 0.000054-20.9$

$0.281048 \pm 0.000071-3.0$

$0.282226 \pm 0.000076-1.5$

$0.281859 \pm 0.000041-0.7$

$0.282020 \pm 0.000037 \quad-28.9$

$0.281081 \pm 0.000064-1.8$

$0.280763 \pm 0.000038 \quad-9.6$

$0.282494 \pm 0.000044-23.9$

$0.282204 \pm 0.000049-1.3$

$0.282144 \pm 0.000027-10.8$

$0.282502 \pm 0.000034-23.1$

$0.282082 \pm 0.000036-13.0$

$0.282358 \pm 0.000043-29.0$

$0.281919 \pm 0.000064-18.4$

$0.282417 \pm 0.000049-4.4$

$0.282126 \pm 0.000030-18.1$

$0.282082 \pm 0.000053-10.3$

$0.281826 \pm 0.000051-4.6$

$0.281574 \pm 0.000061-13.0$

$0.282087 \pm 0.000045-4.3$

$0.282162 \pm 0.000026-2.1$

$0.281715 \pm 0.000039-8.0$

$0.282113 \pm 0.000043 \quad-8.4$

$0.282693 \pm 0.000036-18.9$

$0.282613 \pm 0.000050-14.7$

$0.282114 \pm 0.000037 \quad-7.0$

$0.282441 \pm 0.000039-17.2$
$1376 \quad 1620$

$1657 \quad 1874$

$1526 \quad 1656$

$1784 \quad 1887$

$1807 \quad 2061$

$1511 \quad 1628$

$1357 \quad 2142$

$2726 \quad 2726$

$3114 \quad 3452$

$976 \quad 1535$

$2801 \quad 2871$

$1312 \quad 1349$

$1770 \quad 1787$

$1815 \quad 2556$

$2750 \quad 2793$

$3204 \quad 3420$

$1173 \quad 1805$

$1340 \quad 1372$

$1498 \quad 1770$

$1154 \quad 1768$

$1596 \quad 1924$

$1392 \quad 2157$

$1848 \quad 2310$

$1586 \quad 2047$

$1572 \quad 1830$

$1846 \quad 1959$

$2232 \quad 2548$

$1515 \quad 1622$

$1399 \quad 1451$

$2014 \quad 2209$

$1516 \quad 1726$

$872 \quad 1382$

$935 \quad 1327$

$1504 \quad 1679$

$1177 \quad 1626$ 


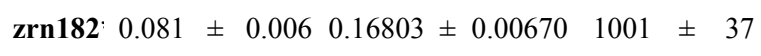
$\begin{array}{lllllll}\operatorname{zrn183} & 0.108 & \pm & 0.005 & 0.16768 \pm 0.00599 & 1768 & \pm 87\end{array}$ zrn184 $0.088 \pm 0.013 \quad 0.18132 \pm 0.00714 \quad 1380 \pm 292$ $\begin{array}{lllllll}\operatorname{zrn185} & 0.159 & \pm & 0.006 & 0.41219 \pm 0.01478 & 2450 & \pm\end{array}$

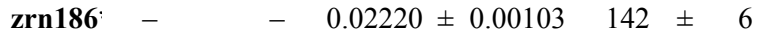
$\operatorname{zrn187} 0.104 \pm 0.003 \quad 0.29019 \pm 0.00906 \quad 1701 \pm 50$

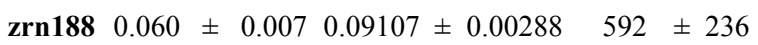
zrn189 $0.090 \pm 0.0030 .10185 \pm 0.003121430 \pm 55$

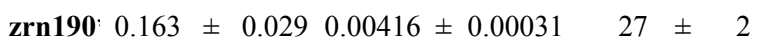
$\begin{array}{lllllll}\text { zrn191 } & 0.164 & \pm & 0.005 & 0.27245 \pm 0.00970 & 2502 & \pm\end{array}$

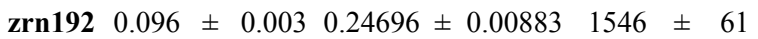
$\begin{array}{lllllll}\operatorname{zrn193} & 0.067 & \pm & 0.010 & 0.07777 \pm 0.00355 & 483 & \pm 21\end{array}$ $\begin{array}{llllll}\text { zrn194: } & 0.064 \pm 0.007 & 0.01189 \pm 0.00044 & 76 & \pm\end{array}$ zrn195 $0.238 \pm 0.0040 .51227 \pm 0.01276 \quad 3105 \pm 28$ zrn196 $0.248 \pm 0.0320 .00329 \pm 0.00021 \quad 21 \pm 1$

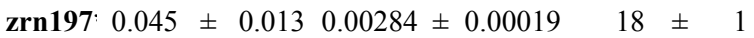
$\begin{array}{lllllll}\text { zrn198} & 0.051 & \pm & 0.005 & 0.06419 \pm 0.00207 & 401 & \pm\end{array}$

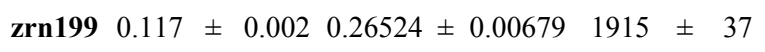
$\begin{array}{lllllll}\text { zrn200 } & 0.077 & \pm & 0.004 & 0.15875 & \pm 0.00463 \quad 1131 & \pm 107\end{array}$ $\begin{array}{llllll}\text { zrn201 } & 0.056 & \pm 0.005 & 0.01209 \pm 0.00039 & 77 & \pm 2\end{array}$ zrn202 $0.103 \pm 0.003 \quad 0.28572 \pm 0.00748 \quad 1681 \pm 49$ zrn203 $0.132 \pm 0.0060 .35806 \pm 0.010602121 \pm 73$ $\begin{array}{llllll}\text { zrn204 } & 0.161 & \pm 0.003 & 0.20232 \pm 0.00521 & 2467 & \pm 35\end{array}$ $\operatorname{zrn205} 0.111 \pm 0.003 \quad 0.29156 \pm 0.00619 \quad 1817 \pm 48$ $\begin{array}{llllll}\text { zrn206 } & 0.063 & \pm 0.003 & 0.01397 \pm 0.00032 & 697 & \pm 109\end{array}$ zrn207 $0.033 \pm 0.010 \quad 0.01160 \pm 0.00071 \quad 74 \pm 5$

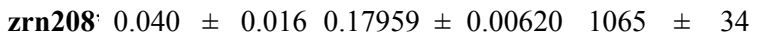
$\begin{array}{lllllll}\text { zrn209 } & 0.053 & \pm 0.008 & 0.02790 & \pm 0.00116 & 177 & \pm\end{array}$

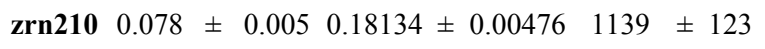
zrn211 $0.173 \pm 0.0020 .52243 \pm 0.01027 \quad 2584 \pm 21$ $\begin{array}{llllllll}\text { zrn212 } & 0.039 & \pm & 0.008 & 0.00641 \pm 0.00027 & 41 & \pm & 2\end{array}$ $\operatorname{zrn213} 0.183 \pm 0.003 \quad 0.49501 \pm 0.01010 \quad 2680 \pm 28$ $\operatorname{zrn214} 0.095 \pm 0.001 \quad 0.27881 \pm 0.00546 \quad 1524 \pm 27$ $\begin{array}{lllllll}\operatorname{zrn} 215 & 0.044 & \pm & 0.004 & 0.01148 \pm 0.00038 & 74 & \pm\end{array}$ zrn216 $0.101 \pm 0.0040 .19766 \pm 0.00358 \quad 1646 \pm 65$

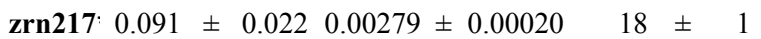
zrn218 $0.103 \pm 0.003 \quad 0.26236 \pm 0.00430 \quad 1685 \pm 47$
$0.282275 \pm 0.000036-7.5$ $0.281580 \pm 0.000054-11.6$ $0.282128 \pm 0.000058 \quad-2.6$ $0.281153 \pm 0.000052-8.2$ $0.282615 \pm 0.000032-18.0$ $0.281946 \pm 0.000056 \quad-0.4$ $0.282134 \pm 0.000038-23.3$ $0.281741 \pm 0.000039-14.9$ $0.282490 \pm 0.000034-25.4$ $0.281178 \pm 0.000032-5.9$ $0.281846 \pm 0.000028 \quad-8.1$ $0.282423 \pm 0.000043-15.9$ $0.282382 \pm 0.000043-28.0$ $0.280546 \pm 0.000075-11.7$ $0.282654 \pm 0.000065-19.8$ $0.282476 \pm 0.000029-26.1$ $0.282597 \pm 0.000045 \quad-11.9$ $0.281329 \pm 0.000054-16.5$ $0.282163 \pm 0.000031-8.0$ $0.282949 \pm 0.000028 \quad-7.9$ $0.281651 \pm 0.000031-11.4$ $0.281446 \pm 0.000032-6.8$ $0.280754 \pm 0.000056-21.9$ $0.281698 \pm 0.000020 \quad-6.1$ $0.282342 \pm 0.000032-13.1$

$0.282214 \pm 0.000056 \quad-7.9$ $0.282612 \pm 0.000044-17.2$ $0.282141 \pm 0.000038 \quad-8.5$ $0.280948 \pm 0.000027-11.8$ $0.282562 \pm 0.000041 \quad-22.5$ $0.281090 \pm 0.000050 \quad-4.1$ $0.281840 \pm 0.000049 \quad-8.9$ $0.282855 \pm 0.000044-11.3$ $0.281951 \pm 0.000048 \quad-1.7$ $0.282846 \pm 0.000025-13.1$ $0.281649 \pm 0.000046-11.4$
Iizuka et al., Table DR1 p.6

$\begin{array}{rrrrrr}1346 & 1538 & 0.282274 \pm 0.000036 & -6.5 & 1296 & 1462 \\ 2291 & 2572 & 0.281578 \pm 0.000054 & -9.9 & 2201 & 2439 \\ 1497 & 1564 & 0.282126 \pm 0.000058 & -1.3 & 1439 & 1471 \\ 2816 & 3006 & 0.281148 \pm 0.000052 & -5.8 & 2699 & 2835 \\ 995 & 1470 & 0.282614 \pm 0.000032 & -18.0 & 964 & 1445 \\ 1719 & 1728 & 0.281944 \pm 0.000056 & 1.3 & 1701 & 1701 \\ 1676 & 2268 & 0.282133 \pm 0.000038 & -22.8 & 1618 & 2203 \\ 2114 & 2480 & 0.281737 \pm 0.000039 & -13.7 & 2035 & 2371 \\ 1228 & 1894 & 0.282490 \pm 0.000034 & -25.5 & 1189 & 1868 \\ 2766 & 2903 & 0.281169 \pm 0.000032 & -3.6 & 2655 & 2739 \\ 1915 & 2116 & 0.281844 \pm 0.000028 & -6.6 & 1840 & 2002 \\ 1229 & 1640 & 0.282422 \pm 0.000043 & -15.6 & 1187 & 1593 \\ 1394 & 2125 & 0.282382 \pm 0.000043 & -28.0 & 1352 & 2090 \\ 3618 & 3882 & 0.280542 \pm 0.000075 & -8.6 & 3467 & 3657 \\ 958 & 1480 & 0.282654 \pm 0.000065 & -19.9 & 930 & 1463 \\ 1253 & 1938 & 0.282476 \pm 0.000029 & -26.2 & 1216 & 1912 \\ 958 & 1272 & 0.282597 \pm 0.000045 & -11.6 & 928 & 1235 \\ 2659 & 3052 & 0.281326 \pm 0.000054 & -14.7 & 2554 & 2904 \\ 1499 & 1702 & 0.282162 \pm 0.000031 & -6.9 & 1439 & 1616 \\ 453 & 668 & 0.282949 \pm 0.000028 & -8.0 & 443 & 660 \\ 2198 & 2477 & 0.281649 \pm 0.000031 & -9.8 & 2113 & 2350 \\ 2425 & 2588 & 0.281444 \pm 0.000032 & -4.7 & 2324 & 2436 \\ 3441 & 3940 & 0.280707 \pm 0.000057 & -21.0 & 3365 & 3844 \\ 2092 & 2242 & 0.281695 \pm 0.000040 & -4.3 & 2008 & 2112 \\ 1308 & 1648 & 0.282340 \pm 0.000032 & -12.6 & 1265 & 1591 \\ & & & & & \\ 1431 & 1634 & 0.282212 \pm 0.000056 & -7.0 & 1379 & 1555 \\ 990 & 1443 & 0.282612 \pm 0.000044 & -17.1 & 959 & 1416 \\ 1533 & 1750 & 0.282139 \pm 0.000038 & -7.5 & 1476 & 1664 \\ 3109 & 3381 & 0.280945 \pm 0.000027 & -9.2 & 2978 & 3188 \\ 1106 & 1697 & 0.282562 \pm 0.000041 & -22.6 & 1072 & 1674 \\ 2864 & 2960 & 0.281086 \pm 0.000050 & -1.5 & 2741 & 2773 \\ 1930 & 2149 & 0.281837 \pm 0.000049 & -7.5 & 1856 & 2038 \\ 610 & 915 & 0.282855 \pm 0.000044 & -11.4 & 595 & 902 \\ 1723 & 1765 & 0.281950 \pm 0.000048 & -0.1 & 1651 & 1653 \\ 638 & 988 & 0.282846 \pm 0.000025 & -13.2 & 623 & 981 \\ 2201 & 2478 & 0.281647 \pm 0.000046 & -9.7 & 2114 & 2349\end{array}$


$\operatorname{zrn219} 0.082 \pm 0.005 \quad 0.15061 \pm 0.00347 \quad 1238 \pm 120$ $\begin{array}{lllllll}\operatorname{zrn220} & 0.141 & \pm & 0.003 & 0.18916 \pm 0.00298 & 2244 & \pm 36\end{array}$

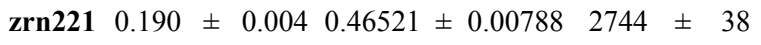

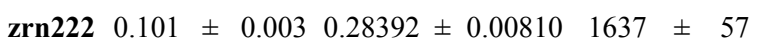
$\operatorname{zrn223} 0.083 \pm 0.004 \quad 0.21766 \pm 0.00680 \quad 1274 \quad \pm 105$ $\begin{array}{llllll}\text { zrn224 } & 0.101 & \pm 0.003 & 0.29809 & \pm 0.00857 \quad 1643 \pm 60\end{array}$ zrn225 $0.094 \pm 0.008 \quad 0.07133 \pm 0.00216 \quad 1511 \pm 168$

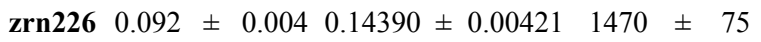
$\begin{array}{lllllll}\operatorname{zrn227} & 0.054 & \pm 0.004 & 0.06534 & \pm 0.00204 & 408 & \pm 12\end{array}$ $\begin{array}{lllllll}\operatorname{zrn} 228 & 0.094 & \pm & 0.003 & 0.19721 \pm 0.00544 & 1515 & \pm 68\end{array}$

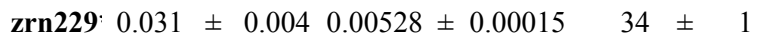
$\begin{array}{llllll}\operatorname{zrn230} & 0.072 & \pm 0.002 & 0.18070 \pm 0.00319 & 979 & \pm 65\end{array}$ $\begin{array}{llllll}\operatorname{zrn} 231 & 0.108 & \pm 0.002 & 0.31441 \pm 0.00503 \quad 1759 & \pm 27\end{array}$

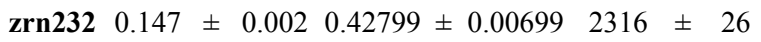
$\begin{array}{lllllll}\text { zrn233 } & 0.103 & \pm & 0.003 & 0.28861 & \pm 0.00553 \quad 1671 & \pm 62\end{array}$ $\begin{array}{lllllll}\text { zrn234 } & 0.027 & \pm & 0.005 & 0.01249 & \pm 0.00044 \quad 80 & \pm 3\end{array}$ $\begin{array}{lllllll}\operatorname{zrn} 235 & 0.076 & \pm & 0.002 & 0.23062 \pm 0.00411 & 1086 & \pm 63\end{array}$ $\begin{array}{lllllll}\text { zrn236 } & 0.050 & \pm & 0.002 & 0.02710 \pm 0.00047 & 184 & \pm 90\end{array}$ $\begin{array}{lllllll}\operatorname{zrn237} & 0.047 & \pm & 0.002 & 0.01276 \pm 0.00023 & 68 & \pm 113\end{array}$

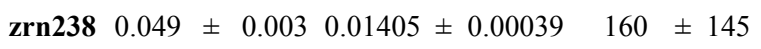
zrn239 $0.096 \pm 0.001 \quad 0.31756 \pm 0.00767 \quad 1555 \pm 21$

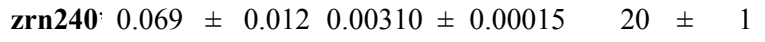
zrn241 $0.084 \pm 0.0020 .23477 \pm 0.00593 \quad 1300 \pm 52$ $\begin{array}{lllllll}\text { zrn242 } & 0.079 & \pm & 0.015 & 0.00364 \pm 0.00021 & 23 & \pm\end{array}$

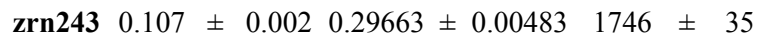
$\operatorname{zrn244} 0.102 \pm 0.002 \quad 0.28859 \pm 0.00481 \quad 1662 \pm 41$

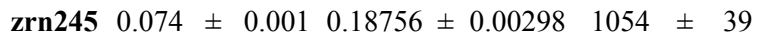
$\operatorname{zrn246} 0.074 \pm 0.001 \quad 0.18035 \pm 0.00392 \quad 1052 \pm 39$ $\begin{array}{llllll}\operatorname{zrn} 247 & 0.088 & \pm 0.002 & 0.24096 \pm 0.00538 & 1385 & \pm 46\end{array}$ $\operatorname{zrn248} 0.071 \pm 0.0020 .16768 \pm 0.00366 \quad 968 \pm 43$ $\operatorname{zrn249} 0.081 \pm 0.003 \quad 0.30001 \pm 0.00659 \quad 1225 \pm 75$

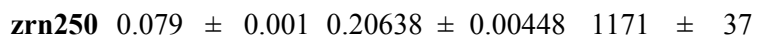
$\operatorname{zrn251} 0.101 \pm 0.0020 .29525 \pm 0.00638 \quad 1638 \pm 28$

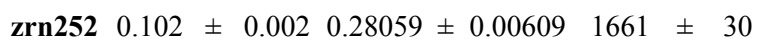

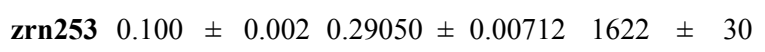
$\begin{array}{lllllllll}\text { zrn254 } & 0.073 & \pm & 0.003 & 0.17446 & \pm 0.00458 & 1005 & \pm & \end{array}$ zrn255 $0.093 \pm 0.0030 .26343 \pm 0.00683 \quad 1479 \pm 58$
$0.282307 \pm 0.000039 \quad 0.0$ $0.280945 \pm 0.000044-21.2$ $0.281026 \pm 0.000029$ $0.281756 \pm 0.000043$ $0.282055 \pm 0.000042$ $0.281917 \pm 0.000043$ $0.281379 \pm 0.000023-25.6$ $0.282013 \pm 0.000031-4.2$ $0.282059 \pm 0.000035-30.7$ $0.281788 \pm 0.000044-11.0$ $0.282129 \pm 0.000046-38.0$ $0.282116 \pm 0.000024-13.7$ $0.281346 \pm 0.000101-20.1$ $0.281210 \pm 0.000058 \quad-9.8$ $0.281905 \pm 0.000039 \quad-2.7$ $0.282748 \pm 0.000048-14.9$ $0.282036 \pm 0.000058-13.7$ $0.282455 \pm 0.000049-22.6$ $0.282858 \pm 0.000054-11.4$ $0.282590 \pm 0.000035-18.5$ $0.281773 \pm 0.000040-10.5$ $0.282456 \pm 0.000054-26.8$ $0.282088 \pm 0.000060-6.1$ $0.282826 \pm 0.000042-13.7$ $0.281250 \pm 0.000054 \quad-23.9$ $0.281849 \pm 0.000062 \quad-4.9$ $0.282058 \pm 0.000056-13.8$

$0.282094 \pm 0.000043 \quad-3.6$ $0.282193 \pm 0.000027-11.2$ $0.281932 \pm 0.000032-13.6$ $0.282093 \pm 0.000028 \quad-9.4$ $0.281831 \pm 0.000024-6.2$ $0.281467 \pm 0.000023-18.5$ $0.281725 \pm 0.000050-10.4$ $0.282099 \pm 0.000061-13.6$ $0.282108 \pm 0.000033 \quad-0.6$

Iizuka et al., Table DR1 p.7

1241

3190

2950

2039

1640

1779

2672

1663

1842

2017

1818

1610

2667

2755

1792

789

1715

1249

606

1032

2032

1286

1583

673

2823

1885

1688

1552

1487

1851

1602

1919

2499

2093

1631

1508
1243

3684

3057

2257

1842

1852

3291

1770

2627

2289

2800

1958

3147

2986

1857

1185

2061

1839

914

1517

2291

1988

1736

1035

3392

2007

2036

1645

1773

2192

1839

2069

2943

2346

1976

1526
$0.282306 \pm 0.000039 \quad 1.1$ $0.280939 \pm 0.000044-19.1$ $0.281022 \pm 0.000029-1.9$ $0.281756 \pm 0.000043 \quad-7.2$ $0.282054 \pm 0.000042-6.8$ $0.281915 \pm 0.000043-1.4$ $0.281374 \pm 0.000023-24.3$ $0.282004 \pm 0.000031-3.1$ $0.282059 \pm 0.000035-30.4$ $0.281785 \pm 0.000044 \quad-9.6$ $0.282129 \pm 0.000046-38.1$ $0.282115 \pm 0.000024-12.8$ $0.281345 \pm 0.000101-18.4$ $0.281210 \pm 0.000058 \quad-7.4$ $0.281904 \pm 0.000039-1.0$ $0.282748 \pm 0.000048-15.0$ $0.282033 \pm 0.000058-12.7$ $0.282454 \pm 0.000049-22.5$ $0.282857 \pm 0.000054-11.4$ $0.282590 \pm 0.000035-18.4$ $0.281769 \pm 0.000040-9.1$ $0.282456 \pm 0.000054-26.9$ $0.282087 \pm 0.000060 \quad-4.9$ $0.282826 \pm 0.000042-13.7$ $0.281249 \pm 0.000054-22.2$ $0.281847 \pm 0.000062-3.3$ $0.282057 \pm 0.000056-12.8$

$0.282092 \pm 0.000043 \quad-2.3$ $0.282193 \pm 0.000027-10.3$ $0.281929 \pm 0.000032-12.6$ $0.282089 \pm 0.000028 \quad-8.4$ $0.281829 \pm 0.000024-4.6$ $0.281464 \pm 0.000023-16.9$ $0.281723 \pm 0.000050-8.8$ $0.282099 \pm 0.000061-12.7$ $0.282106 \pm 0.000033 \quad 0.8$
1238

$3068 \quad 3510$

$2823 \quad 2866$

$1956 \quad 2131$

$1577 \quad 1745$

$1706 \quad 1739$

$2575 \quad 3163$

$1780 \quad 2564$

$1940 \quad 2174$

$1762 \quad 2759$

$1553 \quad 1875$

$2561 \quad 3001$

$2636 \quad 2810$

$1718 \quad 1741$

$765 \quad 1168$

$1655 \quad 1973$

$1209 \quad 1803$

$592 \quad 902$

$999 \quad 1490$

$1955 \quad 2174$

$1248 \quad 1960$

$1519 \quad 1642$

$654 \quad 1026$

$2711 \quad 3241$

$1808 \quad 1887$

$1626 \quad 1946$

$1491 \quad 1550$

$1432 \quad 1695$

$1784 \quad 2095$

$1546 \quad 1757$

$1841 \quad 1951$

$2401 \quad 2810$

$2011 \quad 2225$

$1572 \quad 1890$

$1479 \quad 1479$ 
Iizuka et al., Table DR1 p.8

\begin{tabular}{|c|c|c|c|c|c|c|c|c|c|c|c|c|c|c|}
\hline 56 & 048 & , & 0 & \pm & 50 & \pm & $4 \pm$ & -4.8 & 77 & 0 & $84 \pm 0$ & -4.8 & 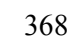 & 499 \\
\hline n257 & 0.071 & ( & 0.002 & $0.16615 \pm 0.00299$ & 969 & \pm 68 & $0.282194 \pm 0.000058$ & -11.2 & 1485 & 1770 & $0.282193 \pm 0.000058$ & -10.3 & 1431 & 1693 \\
\hline n258 & 0.074 & 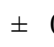 & 0.002 & $0.15978 \pm 0.00274$ & 32 & \pm 55 & $282178 \pm 0.000068$ & -10.1 & 96 & 753 & $282178 \pm 0.000068$ & -9.1 & $H$ & 73 \\
\hline n259: & 0.045 & ( & 0.009 & $0710 \pm 0.00034$ & 46 & \pm & $282235 \pm 0.0$ & -34.0 & 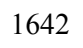 & 23 & $235 \pm 0$ & -34.0 & 2 & 84 \\
\hline n260 & 198 & \pm & 0.003 & $54376 \pm 0.00898$ & 813 & \pm 24 & $3 \pm 0$ & -8 & 3191 & 6 & $59 \pm 1$ & -5.7 & 5 & 3184 \\
\hline n261 & 099 & , & 0.002 & $10 \pm 0$ & 601 & $\pm \quad 42$ & & & & & & & & \\
\hline n262 & 110 & 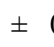 & 0.003 & $6 \pm 0$ & 06 & \pm 44 & & & & & & & & \\
\hline n263 & 0.086 & 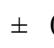 & 0.001 & $6=0.0$ & 343 & \pm 31 & $80 \pm 0.000053$ & -5.2 & 584 & 1717 & $080 \pm 0.0$ & -3.9 & 1520 & 1619 \\
\hline 4 & 0.056 & ( & 0.008 & $69 \pm 0$ & 30 & \pm & $34 \pm 0$ & -32.6 & 565 & 2413 & $84 \pm 0$ & -32.7 & 516 & 2377 \\
\hline 5 & 100 & 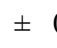 & 0.002 & $32 \pm 0.0$ & 617 & \pm 31 & $725 \pm$ & -10.5 & 2094 & 2352 & $23 \pm 0$ & -9.0 & 2012 & 228 \\
\hline 6 & 0.104 & \pm & 0.023 & \pm 0.0 & 21 & \pm & $540 \pm$ & -23.8 & 148 & 1774 & $540 \pm 0$ & -23.9 & 113 & 1752 \\
\hline 67 & 0.055 & \pm & 0.003 & $0.06140 \pm 0.00124$ & 432 & \pm 131 & $281868 \pm 0.000042$ & -36.8 & 145 & 3078 & $868 \pm 0.0$ & -36.5 & 2072 & 006 \\
\hline 8 & 0.084 & \pm & 0.002 & $73 \pm 0.00310$ & 1286 & \pm 40 & 066 & -2.9 & 421 & 1497 & 066 & -1.7 & 365 & 409 \\
\hline 269 & 0.109 & \pm & 0.002 & \pm 0.00573 & 1787 & $\pm \quad 39$ & $281587 \pm$ & -10.8 & 2276 & 2538 & $0.281586 \pm 0$ & -9.0 & 2184 & 399 \\
\hline & 0.130 & \pm & 0.004 & \pm 0.00207 & 2092 & \pm 51 & $281478 \pm c$ & -6.4 & 2381 & 2536 & $476 \pm$ & -4.3 & 2283 & 382 \\
\hline 71 & 0.085 & \pm & 0.002 & $0.21069 \pm 0.00229$ & 1319 & \pm 36 & $0.282108 \pm$ & -4.9 & 1544 & 1668 & $82106 \pm 0$ & -3.7 & 35 & 579 \\
\hline 72 & 0.077 & \pm & 0.003 & $=0.00256$ & 1132 & \pm 83 & $0.282193 \pm$ & -6.9 & 1450 & 1621 & $92 \pm$ & -5.8 & 1394 & 541 \\
\hline 3 & 0.101 & \pm & 0.002 & 394 & 649 & \pm 45 & $4 \pm$ & -6.5 & 1944 & 03 & $2 \pm$ & -4.9 & 866 & 1984 \\
\hline 74 & 0.056 & \pm & 0.005 & \pm 0.00023 & 64 & \pm & $6 \pm$ & -34.9 & 1701 & 2607 & $96 \pm 1$ & -34.9 & 1650 & 562 \\
\hline 275 & 0.051 & \pm & 0.006 & $0.00389 \pm 0.00011$ & 25 & \pm & $0.282741 \pm$ & -16.6 & 81 & 1252 & $741 \pm 0$ & -16.7 & 0 & 0 \\
\hline n276 & 0.088 & \pm & 0.002 & $0.23336 \pm 0.00299$ & 384 & \pm 54 & $0.282061 \pm 0.000054$ & -4.8 & 1606 & 1729 & $0.282059 \pm 0$ & -3.5 & 542 & 630 \\
\hline n277: & 0.051 & \pm & 0.006 & $0.01047 \pm 0.00031$ & 67 & \pm & $0.282161 \pm 0.000050$ & -36.0 & 1758 & 2692 & $0.282161 \pm 0$ & -36.1 & 1704 & 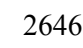 \\
\hline n278 & 0.075 & \pm & 0.002 & $0.19860 \pm 0.00325$ & 1080 & \pm 63 & $0.282308 \pm 0.000025$ & -4 & 1274 & 1383 & $0.282307 \pm 0$ & -3.2 & 1224 & 1307 \\
\hline n279 & 0.069 & \pm & 0.004 & $0.15172 \pm 0.00237$ & 891 & \pm 114 & $0.282355 \pm 0.000075$ & -7.6 & 1241 & 1436 & $0.282351 \pm 0$ & -6.9 & 1199 & 376 \\
\hline n280 & 0.113 & \pm & 0.002 & \pm 0.0 & 850 & \pm 35 & $281652 \pm c$ & -6 & 215 & 2323 & $51 \pm$ & -4.9 & 20 & 2184 \\
\hline 81 & 0.092 & \pm & 0.002 & $7 \pm 0$ & 457 & \pm 50 & $31928 \pm 0$ & -7.6 & 80 & 1993 & $27 \pm 0$ & -6.2 & 732 & 1884 \\
\hline ? & 0.097 & \pm & 0.002 & \pm 0.0 & 1576 & \pm 45 & $281853 \pm$ & -7.1 & 1897 & 2073 & $851 \pm 0$ & -5.6 & 1823 & 958 \\
\hline 283 & 0.087 & \pm & 0.002 & $0.25857 \pm 0.00396$ & 1368 & \pm 36 & $0.281891 \pm$ & -11.3 & 1884 & 2166 & $0.281890 \pm 0.000029$ & -10.0 & 1812 & 2058 \\
\hline 84 & 0.098 & \pm & 0.009 & $0.34447 \pm 0.00717$ & 1580 & \pm 171 & $0.281429 \pm 0.000052$ & -22.0 & 2577 & 3107 & 0.281428 & -20.4 & 2476 & 1 \\
\hline & .044 & \pm & 0.004 & $0.00855 \pm 0.00019$ & 55 & \pm & & & & & & & & \\
\hline 286 & 0.079 & \pm & 0.003 & $0.18888 \pm 0.00263$ & 175 & \pm 87 & $32 \pm$ & -15.0 & 1866 & 2238 & 932 & -13.9 & 1792 & 136 \\
\hline 287 & 0.087 & \pm & 0.002 & $0.22580 \pm 0.00336$ & 1359 & $\pm \quad 39$ & $0.281969 \pm 0.000060$ & -8.8 & 1760 & 1980 & $0.281967 \pm 0.000060$ & -7.5 & 1693 & 1879 \\
\hline 38 & 0.046 & \pm & 0.004 & $0.01414 \pm 0.00033$ & 91 & \pm & $0.282479 \pm 0.000044$ & -24.2 & 1231 & 1866 & $0.282479 \pm 0.000044$ & -24.2 & 1193 & 836 \\
\hline 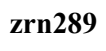 & 0.074 & \pm & 0.002 & $0.16067 \pm 0.00245$ & 1030 & \pm 50 & $0.282069 \pm 0.000026$ & -14.0 & 1675 & 2029 & $0.282069 \pm 0.000026$ & -13.0 & 1613 & 941 \\
\hline & 0.076 & \pm & 0.002 & $0.16616 \pm 0.00274$ & 1096 & \pm 59 & $0.282096 \pm 0.000024$ & -11.3 & 1615 & 1901 & $0.282095 \pm 0.000024$ & -10.2 & 1555 & 4 \\
\hline & 0.084 & \pm & 0.003 & $0.25613 \pm 0.00463$ & 1289 & \pm 70 & $0.281869 \pm 0.000034$ & -14.2 & 1938 & 2292 & $0.281868 \pm 0.000034$ & -13.0 & 1865 & 4 \\
\hline & 0.066 & 工 & 0.002 & $0.14762 \pm 0.00242$ & 822 & \pm 64 & $0.282236 \pm$ & -13.6 & 1451 & 1800 & $0.282236 \pm 0.000031$ & -12.9 & 1401 & 1730 \\
\hline
\end{tabular}


$\operatorname{zrn293} 0.085 \pm 0.003 \quad 0.22312 \pm 0.00378 \quad 1310 \pm 57$

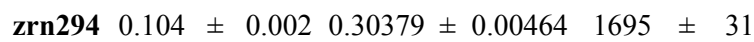
zrn295 $0.096 \pm 0.006 \quad 0.27922 \pm 0.00512 \quad 1544 \pm 122$ zrn296 $0.086 \pm 0.001 \quad 0.20505 \pm 0.00308 \quad 1332 \pm 32$

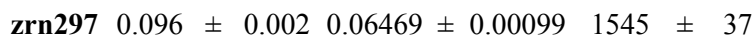
zrn298 $0.078 \pm 0.0020 .18620 \pm 0.00288 \quad 1156 \pm 41$ zrn299 $0.162 \pm 0.0020 .36901 \pm 0.005472476 \pm 20$ $\begin{array}{llllll}\text { zrn300 } & 0.039 & \pm 0.016 & 0.00040 \pm 0.00003 & 3 & \pm 0\end{array}$

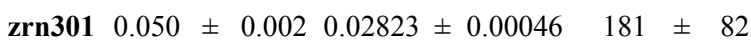
zrn302 $0.085 \pm 0.0020 .05663 \pm 0.00092 \quad 1324 \pm 53$ $\begin{array}{llllll}\text { zrn303 } & 0.047 & \pm 0.005 & 0.02418 & \pm 0.00077 \quad 154 & \pm 5\end{array}$ $\begin{array}{lllllll}\text { zrn304 } & - & - & 0.00922 \pm 0.00108 & 59 & \pm & 7\end{array}$ $\begin{array}{llllll}\operatorname{zrn305} & 0.039 & \pm 0.011 & 0.01183 \pm 0.00068 & 76 & \pm\end{array}$ $\begin{array}{llllll}\text { zrn306 } & 0.089 & \pm 0.003 & 0.23408 \pm 0.00566 & 1409 & \pm 69\end{array}$ $\begin{array}{lllllll}\text { zrn307 } & 0.036 & \pm 0.006 & 0.00341 \pm 0.00013 \quad 22 & \pm & 1\end{array}$ zrn308 $0.186 \pm 0.003 \quad 0.49976 \pm 0.011592706 \pm 31$ $\begin{array}{llllll}\text { zrn309} & 0.041 & \pm 0.005 & 0.00951 \pm 0.00030 \quad 61 & \pm 2\end{array}$ $\begin{array}{llllll}\text { zrn310 } & 0.112 \pm 0.003 & 0.30663 \pm 0.00716 \quad 1825 & \pm 48\end{array}$

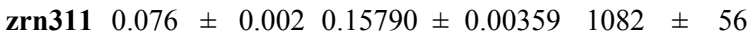
zrn312 $0.077 \pm 0.003 \quad 0.19382 \pm 0.00468 \quad 1126 \pm 75$ $\begin{array}{lllllll}\text { zrn313 } & 0.053 & \pm 0.004 & 0.02017 & \pm 0.00054 & 129 & \pm\end{array}$

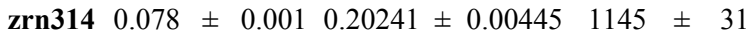
zrn315 $0.090 \pm 0.0060 .13742 \pm 0.00356 \quad 1423 \pm 123$ zrn316 $0.104 \pm 0.0020 .27340 \pm 0.00686 \quad 1692 \pm 39$

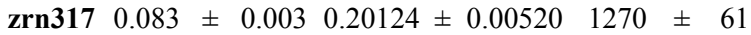

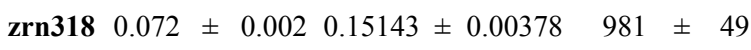

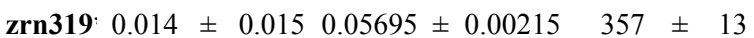

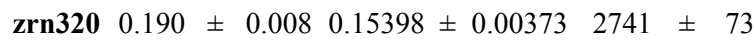
$\begin{array}{llllllll}\text { zrn321 } & 0.108 & \pm & 0.002 & 0.23317 & \pm 0.00531 & 1760 & \pm 40\end{array}$

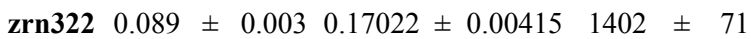

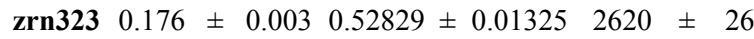

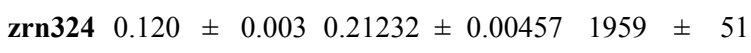

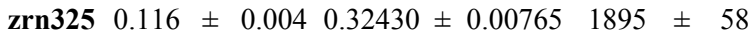

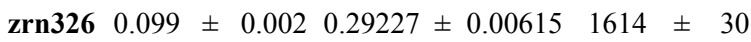
$\begin{array}{lllllll}\text { zrn327 } & 0.072 & \pm & 0.004 & 0.15906 \pm 0.00359 & 986 & \pm 116\end{array}$

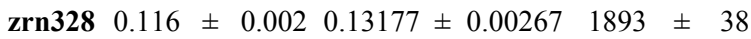
$\begin{array}{lllll}\text { zrn329 } & 0.059 \pm 0.010 & 0.00994 \pm 0.00044 \quad 64 \pm 3\end{array}$
$0.282044+0.000031-7.4$ $0.281851 \pm 0.000026-3.9$ $0.281771 \pm 0.000031-10.8$

$0.282091 \pm 0.000026 \quad-9.9$ $0.280976 \pm 0.000036-13.8$ $0.282880 \pm 0.000078-12.3$

$0.282106 \pm 0.000033-4.8$

$0.282524 \pm 0.000040 \quad-20.9$

$0.282294 \pm 0.000036-31.5$

$0.282629 \pm 0.000026-19.3$

$0.281896 \pm 0.000022-10.0$

$0.282690 \pm 0.000037-18.5$

$0.281038 \pm 0.000020 \quad-5.2$

$0.282610 \pm 0.000043-20.3$

$0.281758 \pm 0.000041-3.7$

$0.282262 \pm 0.000037-5.8$

$0.282152 \pm 0.000041-8.5$

$0.282637 \pm 0.000021-17.6$

$0.281916 \pm 0.000023 \quad-16.4$

$0.281633 \pm 0.000025-18.9$

$0.281834 \pm 0.000032-4.6$

$0.282043 \pm 0.000044 \quad-8.5$

$0.282131 \pm 0.000036-13.1$

$0.282602 \pm 0.000025 \quad-12.9$

$0.281891 \pm 0.000033 \quad-0.8$

$0.282304 \pm 0.000028 \quad 4.3$

$0.281154 \pm 0.000024-3.5$

$0.281441 \pm 0.000021-11.4$

$0.281215 \pm 0.000021-21.1$

$0.281909 \pm 0.000032-4.0$

$0.282115 \pm 0.000022-13.5$

$0.281155 \pm 0.000019-23.3$

$0.283058 \pm 0.000025 \quad-4.4$

Iizuka et al., Table DR1 p.9

1873

2037

1610

3088

1546

1142

1543

987

1867

899

2940

1023

1993

1349

1517

961

1900

2289

1901

1662

1586

963

1794

1402

2777

2472

2844

1798

1609

2940

273
1837

1969

2304

\section{7}

914

\section{0}

1692

2365

1496

2117

1387

3061

1560

2085

1498

1733

1425

2306

2751

2016

1877

1919

1300

1813

1402

2859

2743

3342

1897

1952

3490

395
$0.282043 \pm 0.00003$

$0.281848 \pm 0.000026$

$0.281769 \pm 0.000031$

$-6.2$

$-2.3$

$-9.4$

1586

$1798 \quad 1855$

$1958 \quad 2185$

$0.282089 \pm 0.000026-8.8$

$0.280972 \pm 0.000036-11.3$

1773

$2961 \quad 3224$

$0.282104 \pm 0.000033 \quad-3.6 \quad 1487 \quad 1577$

$0.282523 \pm 0.000040 \quad-20.9 \quad 1106 \quad 1661$

$0.282294 \pm 0.000036 \quad-31.6 \quad 1496 \quad 2324$

$\begin{array}{lllll}0.282629 & \pm 0.000026 & -19.3 & 957 & 1472\end{array}$

$\begin{array}{lllll}0.281895 & \pm 0.000022 & -8.7 & 1795 & 2008\end{array}$

$0.282690 \pm 0.000037 \quad-18.6 \quad 872 \quad 1372$

$0.281036 \pm 0.000020 \quad-2.5 \quad 2810 \quad 2866$

$\begin{array}{lllll}0.282609 & \pm 0.000043 & -20.4 & 992 & 1537\end{array}$

$0.281756 \pm 0.000041 \quad-1.9 \quad 1911 \quad 1958$

$0.282261 \pm 0.000037 \quad-4.8 \quad 1296 \quad 1418$

$0.282151 \pm 0.000041 \quad-7.5 \quad 1461 \quad 1649$

$0.282637 \pm 0.000021 \quad-17.6 \quad 931 \quad 1400$

$0.281914 \pm 0.000023 \quad-15.3 \quad 1828 \quad 2208$

$0.281629 \pm 0.000025 \quad-17.7 \quad 2204 \quad 2636$

$0.281827 \pm 0.000032 \quad-3.1 \quad 1832 \quad 1910$

$0.282041 \pm 0.000044 \quad-7.4 \quad 1599 \quad 1781$

$0.282131 \pm 0.000036-12.2 \quad 1528 \quad 1837$

$0.282602 \pm 0.000025 \quad-12.6 \quad 930 \quad 1265$

$\begin{array}{lllll}0.281889 \pm 0.000033 & 0.9 & 1760 & 1760\end{array}$

$0.282303 \pm 0.000028 \quad 5.6 \quad 1402 \quad 1402$

$0.281151 \pm 0.000024 \quad-0.8 \quad 2655 \quad 2675$

$0.281449 \pm 0.000323 \quad-9.1 \quad 2356 \quad 2571$

$0.281215 \pm 0.000021 \quad-19.2 \quad 2728 \quad 3183$

$0.281908 \pm 0.000032 \quad-2.5 \quad 1725 \quad 1786$

$0.282113 \pm 0.000022 \quad-12.6 \quad 1554 \quad 1874$

$0.281151 \pm 0.000019 \quad-21.5 \quad 2826 \quad 3337$

$0.283058 \pm 0.000025 \quad-4.5 \quad 269 \quad 390$ 


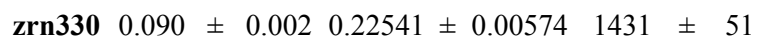
$\begin{array}{lllllll}\text { zrn331 } & 0.095 & \pm & 0.004 & 0.24867 \pm 0.00689 & 1521 & \pm\end{array}$ $\begin{array}{llllll}\text { zrn332 } & 0.075 & \pm & 0.006 & 0.01540 \pm 0.00046 \quad 99 & \pm\end{array}$ $\begin{array}{lllllll}\text { zrn333 } & 0.069 & \pm 0.010 & 0.01060 \pm 0.00044 & 68 & \pm\end{array}$

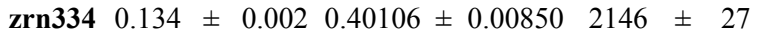

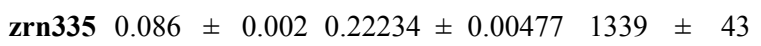
$\begin{array}{lllll}\text { zrn336 } & 0.101 & \pm 0.002 & 0.28919 \pm 0.00633 \quad 1651 & \pm 44\end{array}$ $\begin{array}{llllllll}\operatorname{zrn337} & 0.051 & \pm & 0.003 & 0.04296 & \pm 0.00096 & 258 & \pm 155\end{array}$

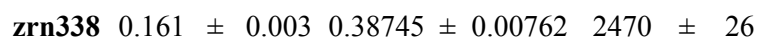
$\begin{array}{llllll}\text { zrn339 } & 0.147 \pm 0.011 & 0.43699 & \pm 0.01154 & 2312 & \pm 133\end{array}$ $\begin{array}{llllll}\text { zrn340 } & 0.075 & \pm 0.006 & 0.07107 & \pm 0.00198 \quad 443 & \pm 12\end{array}$ $\begin{array}{llllllll}\text { zrn341 } & 0.178 & \pm & 0.008 & 0.47661 & \pm 0.01175 & 2633 & \pm\end{array}$ $\begin{array}{llllll}\text { zrn342 } & 0.105 & \pm 0.002 & 0.29710 \pm 0.00588 & 1718 & \pm 34\end{array}$

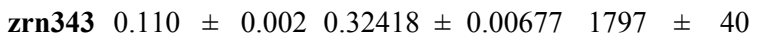
$\begin{array}{llllll}\text { zrn344 } & 0.106 & \pm 0.003 & 0.30321 & \pm 0.00643 \quad 1738 & \pm 45\end{array}$ $\begin{array}{llllll}\text { zrn345 } & 0.179 & \pm 0.004 & 0.44660 \pm 0.00956 & 2644 & \pm 36\end{array}$ $\begin{array}{lllllll}\text { zrn346 } & 0.044 & \pm & 0.003 & 0.01380 \pm 0.00034 & 88 & \pm 2\end{array}$ $\begin{array}{lllllll}\text { zrn347 } & 0.052 & \pm & 0.003 & 0.02692 \pm 0.00062 & 301 & \pm 133\end{array}$

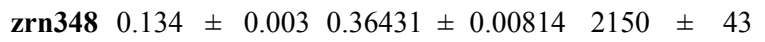
zrn349 $0.090 \pm 0.0020 .23627 \pm 0.00513 \quad 1434 \pm 46$

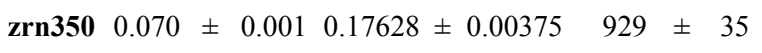

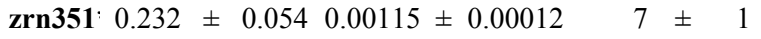
$\begin{array}{llllll}\text { zrn352 } & 0.069 & \pm 0.003 & 0.16488 \pm 0.00478 & 897 & \pm 94\end{array}$ $\begin{array}{lllllll}\text { zrn353 } & 0.104 & \pm & 0.003 & 0.31165 \pm 0.00858 & 1697 & \pm 4\end{array}$

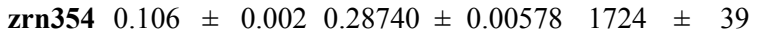

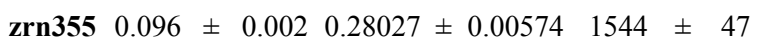

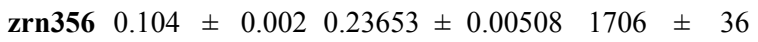
$\begin{array}{llllll}\text { zrn357 } & - & - & 0.00394 \pm 0.00050 & 25 & \pm\end{array}$ zrn358 $0.183 \pm 0.0020 .50406 \pm 0.01082 \quad 2679 \pm 22$

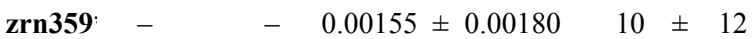

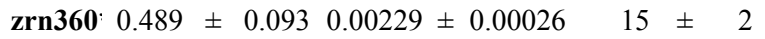

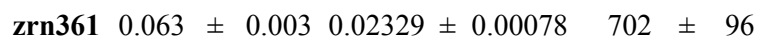

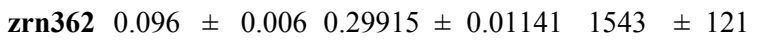

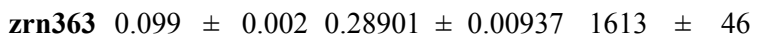
$\begin{array}{llllll}\text { zrn364 } & 0.200 & \pm 0.003 & 0.52862 \pm 0.01702 & 2823 & \pm 28\end{array}$

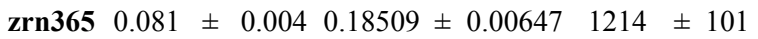
zrn366 $0.075 \pm 0.004 \quad 0.17452 \pm 0.00603 \quad 1080 \pm 100$
$0.281986 \pm 0.000028 \quad-6.2$ $0.282024 \pm 0.000023-2.5$ $0.282527 \pm 0.000041 \quad-22.3$ $0.282437 \pm 0.000044 \quad-26.2$ $0.281442 \pm 0.000038 \quad-6.2$ $0.282033 \pm 0.000029 \quad-7.0$ $0.281815 \pm 0.000046 \quad-6.4$ $0.282632 \pm 0.000031-14.4$ $0.281015 \pm 0.000035-12.6$ $0.280963 \pm 0.000023-18.7$ $0.282495 \pm 0.000042-14.4$ $0.281234 \pm 0.000038 \quad-0.3$ $0.281655 \pm 0.000021-10.3$ $0.281529 \pm 0.000020-12.6$ $0.281603 \pm 0.000016-11.6$ $0.280988 \pm 0.000032 \quad-8.7$ $0.282391 \pm 0.000022 \quad-27.3$ $0.282573 \pm 0.000058-15.3$ $0.281038 \pm 0.000044 \quad-20.5$ $0.282032 \pm 0.000024 \quad-4.5$ $0.281997 \pm 0.000027 \quad-19.2$ $0.282535 \pm 0.000026-24.4$ $0.282112 \pm 0.000022-16.0$ $0.281904 \pm 0.000027 \quad-2.0$ $0.281876 \pm 0.000020 \quad-2.2$ $0.281908 \pm 0.000036-6.0$ $0.281769 \pm 0.000022 \quad-6.5$ $0.282416 \pm 0.000016-28.1$ $0.280902 \pm 0.000017 \quad-10.8$

$0.282597 \pm 0.000064 \quad-22.0$ $0.282510 \pm 0.000059 \quad-7.0$ $0.281815 \pm 0.000021$ $0.281974 \pm 0.000029 \quad-1.8$ $0.280828 \pm 0.000048 \quad-9.5$ $0.282155 \pm 0.000036-6.0$ $0.282273 \pm 0.000038 \quad-5.5$

Iizuka et al., Table DR1 p.10

1634

1149

1305

2425

1661

1941

939

3029

3147

1120

2646

2183

2367

2262

3033

1376

1025

3065

1640

1819

1159

1639

1788

1826

1816

2003

1350

3158

1055

1031

1966

1693

3242

1492

1332
1735

1993

2575

1839

2099

1317

3320

3582

1494

2655

2433

2673

2544

3234

2091

1426

3544

1755

2301

1799

2043

1837

1880

1965

2164

2084

3406

1633

1216

2191

1737

3458

1646

1472
$0.281982+0.000028$ $0.282023 \pm 0.000023$

$\pm 0.000041-22.3$

$0.282437 \pm 0.000044-26.3$

$0.281440 \pm 0.000038-4.1$

$0.282031 \pm 0.000029-5.8$

$0.281813 \pm 0.000046-4.8$

$0.282631 \pm 0.000031-14.3$

$0.281009 \pm 0.000035-10.2$

$0.280961 \pm 0.000023-16.4$

$0.282495 \pm 0.000042-14.1$

$0.281231 \pm 0.000038 \quad 2.4$

$0.281653 \pm 0.000021-8.6$

$0.281527 \pm 0.000020-10.9$

$0.281601 \pm 0.000016-9.9$

$0.280985 \pm 0.000032-6.1$

$0.282391 \pm 0.000022-27.4$

$0.282573 \pm 0.000058-15.1$

$0.281037 \pm 0.000044-18.3$

$0.282030 \pm 0.000024-3.2$

$0.281995 \pm 0.000027 \quad-18.4$

$0.282535 \pm 0.000026-24.5$

$0.282112 \pm 0.000022-15.2$

$0.281900 \pm 0.000027 \quad-0.4$

$0.281874 \pm 0.000020-0.6$

$0.281905 \pm 0.000036-4.5$

$0.281767 \pm 0.000022 \quad-4.9$

$0.282416 \pm 0.000016 \quad-28.2$

$0.280898 \pm 0.000017-8.1$

$0.282508+0.000059$

$0.281972 \pm 0.000029-0.2$

$0.280824 \pm 0.000048 \quad-6.6$

$0.282154 \pm 0.000036 \quad-4.9$

$0.282272 \pm 0.000038-4.4$
$1652 \quad 1774$

$1567 \quad 1591$

$1114 \quad 1706$

$1265 \quad 1962$

$2324 \quad 2417$

$1598 \quad 1743$

$1864 \quad 1982$

$908 \quad 1289$

$2906 \quad 3140$

$3016 \quad 3398$

$1080 \quad 1450$

$2633 \quad 2633$

$2096 \quad 2302$

$2274 \quad 2534$

$2172 \quad 2408$

$2902 \quad 3042$

13332058

$991 \quad 1393$

$2939 \quad 3367$

$1576 \quad 1654$

$1753 \quad 2217$

$1578 \quad 1964$

$1718 \quad 1728$

$1745 \quad 1857$

$1923 \quad 2044$

$1308 \quad 2054$

$3026 \quad 3213$

$1023 \quad 1613$

$997 \quad 1168$

$1888 \quad 2075$

$1624 \quad 1629$

$3104 \quad 3256$

$1432 \quad 1558$

$1279 \quad 1393$ 
$\begin{array}{lllllll}\operatorname{zrn367} & 0.048 & \pm 0.002 & 0.03237 & \pm 0.00090 & 107 & \pm 97\end{array}$

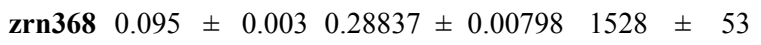

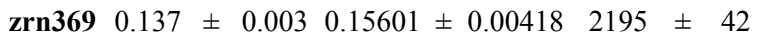
$\begin{array}{lllllll}\text { zrn370 } & 0.051 & \pm 0.005 & 0.01253 \pm 0.00043 \quad 80 \pm 3\end{array}$

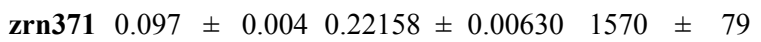
$\operatorname{zrn372} 0.175 \pm 0.003 \quad 0.49314 \pm 0.015752602 \pm 26$ $\begin{array}{llllll}\operatorname{zrn373} & 0.099 & \pm 0.003 & 0.28410 \pm 0.00902 & 1609 & \pm 55\end{array}$

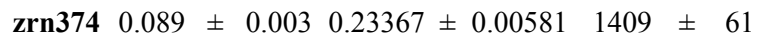
$\begin{array}{lllllll}\operatorname{zrn375} & 0.089 & \pm & 0.002 & 0.24957 \pm 0.00610 & 1398 & \pm 54\end{array}$ $\begin{array}{lllllll}\text { zrn376 } & 0.093 & \pm & 0.019 & 0.00421 \pm 0.00027 & 27 & \pm\end{array}$ $\begin{array}{llllll}\operatorname{zrn377} & 0.106 & \pm 0.003 & 0.28920 \pm 0.00923 \quad 1737 & \pm 56\end{array}$ zrn378 $0.096 \pm 0.005 \quad 0.24716 \pm 0.00814 \quad 1545 \pm 105$ $\begin{array}{lllllll}\text { zrn379 } & 0.106 & \pm 0.015 & 0.00438 & \pm 0.00023 \quad 28 & \pm & 1\end{array}$

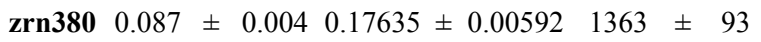
$\begin{array}{llllllll}\operatorname{zrn381} & 0.101 & \pm & 0.004 & 0.27776 & \pm 0.00878 \quad 1651 & \pm 70\end{array}$ $\begin{array}{lllllll}\operatorname{zrn382} & 0.100 & \pm 0.003 & 0.25303 & \pm 0.00787 \quad 1626 & \pm 50\end{array}$ $\begin{array}{lllllll}\text { zrn383 } & 0.076 & \pm 0.008 & 0.18718 & \pm 0.00551 & 1086 & \pm 209\end{array}$

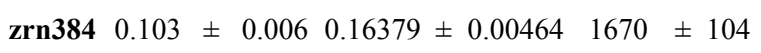
zrn385 $0.076 \pm 0.0020 .16289 \pm 0.00370 \quad 1092 \pm 64$ $\begin{array}{llllll}\text { zrn386 } & 0.120 & \pm 0.088 & 0.00321 \pm 0.00039 & 21 & \pm 2\end{array}$

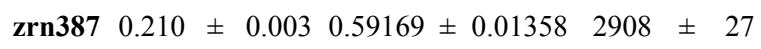
$\begin{array}{lllllll}\text { zrn388 } & 0.175 & \pm & 0.003 & 0.33710 \pm 0.00777 & 2604 & \pm 33\end{array}$ $\begin{array}{lllllll}\text { zrn389 } & 0.075 & \pm & 0.003 & 0.15162 \pm 0.00385 & 1067 & \pm 81\end{array}$

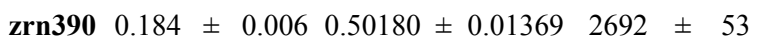
zrn391 $0.234 \pm 0.0060 .58213 \pm 0.01519 \quad 3080 \pm 40$ $\begin{array}{lllllll}\text { zrn392 } & - & - & 0.00271 & \pm 0.00043 \quad 17 & \pm\end{array}$

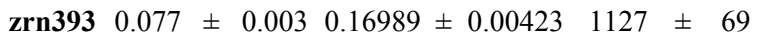
$\begin{array}{llllll}\text { zrn394 } & 0.095 & \pm 0.002 & 0.19940 \pm 0.00469 & 1532 & \pm 46\end{array}$

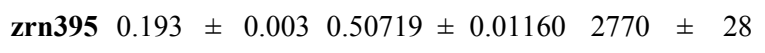
$\begin{array}{lllllll}\text { zrn396 } & 0.091 & \pm 0.002 & 0.24556 & \pm 0.00558 & 1456 & \pm 4\end{array}$ $\begin{array}{lllllll}\text { zrn397 } & 0.108 & \pm 0.003 & 0.14454 & \pm 0.00447 \quad 1760 & \pm 49\end{array}$ $\begin{array}{lllllll}\text { zrn398 } & 0.040 & \pm 0.013 & 0.00567 \pm 0.00039 & 36 & \pm 3\end{array}$ $\begin{array}{lllllll}\text { zrn399: } & 0.060 \pm 0.008 & 0.01452 \pm 0.00066 & 93 & \pm 4\end{array}$

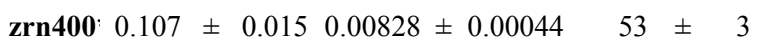

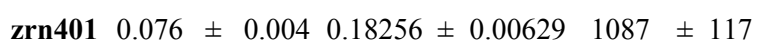

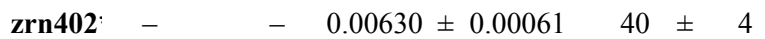

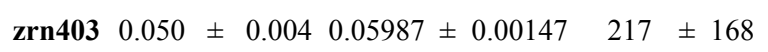

$0.282556 \pm 0.000023-21.0$ $0.281817 \pm 0.000025-9.6$ $0.281072 \pm 0.000025-18.0$ $0.282116 \pm 0.000046-37.2$ $0.281905 \pm 0.000038 \quad-5.4$ $0.280650 \pm 0.000026-21.9$ $0.281730 \pm 0.000049-10.5$ $0.282100 \pm 0.000038 \quad-2.8$

$0.282696 \pm 0.000025-18.2$ $0.281945 \pm 0.000042 \quad 0.6$ $0.281962 \pm 0.000032-4.0$ $0.282459 \pm 0.000031-26.5$ $0.282142 \pm 0.000038 \quad-2.5$ $0.281810 \pm 0.000023 \quad-6.6$ $0.281868 \pm 0.000023 \quad-5.2$ $0.282251 \pm 0.000043 \quad-6.0$ $0.282049 \pm 0.000076 \quad 2.4$ $0.282238 \pm 0.000048$ $0.283006 \pm 0.000043$ $0.280898 \pm 0.000025$ $0.281134 \pm 0.000044$ $0.282146 \pm 0.000042-10.3$

$0.280953 \pm 0.000032 \quad 2.1$ $0.282485 \pm 0.000023 \quad-25.9$ $0.282102 \pm 0.000024-10.3$ $0.281956 \pm 0.000033 \quad-4.6$ $0.280989 \pm 0.000050 \quad-5.2$ $0.282038 \pm 0.000056 \quad-3.7$ $0.281125 \pm 0.000093 \quad-27.9$ $0.282416 \pm 0.000056 \quad-27.8$ $0.282790 \pm 0.000069-13.1$

$0.282143 \pm 0.000025 \quad-9.9$ $0.282004 \pm 0.000069-42.2$ $0.282593 \pm 0.000037 \quad-16.8$

Iizuka et al., Table DR1 p.11

1967

3002

1827

1815

3572

2088

1537

888

1737

1729

1278

1479

1950

1861

1366

1670

1385

370

3115

2813

1541

3080

1239

1599

1741

3003

1626

3020

1347

712

1542

2019

1012
3424

2791

1949

4067

2348

1609

1368

1737

1831

1971

1544

2114

1990

1521

1670

1545

568

3222

2921

1803

3080

1916

1860

1856

3124

1721

3678

2073

1065

1793

3106

1455
$0.282555+0.000023-21.0$ $0.281816 \pm 0.000025-8.1$

$0.281067 \pm 0.000025-15.9$

$0.282116 \pm 0.000046-37.3$

$0.281903 \pm 0.000038-3.9$

$0.280646 \pm 0.000026-19.3$

$0.281727 \pm 0.000049 \quad-9.0$

$0.282098 \pm 0.000038-1.5$

$0.282696 \pm 0.000025-18.2$

$0.281923 \pm 0.000044 \quad 1.5$

$0.281960 \pm 0.000032-2.5$

$0.282459 \pm 0.000031-26.6$

$0.282141 \pm 0.000038-1.2$

$0.281808 \pm 0.000023-5.0$

$0.281866 \pm 0.000023-3.6$

$0.282249 \pm 0.000025 \quad-5.1$

$0.282047 \pm 0.000081$

$0.282237 \pm 0.000048-5.3$

$0.283006 \pm 0.000043-7.4$

$0.280894 \pm 0.000025-1.7$

$0.281129 \pm 0.000044-2.1$

$0.282145 \pm 0.000042$

$0.280925 \pm 0.000030 \quad 4.4$ $0.282485 \pm 0.000023-25.9$ $0.282100+0.000024-9.2$

$0.281952 \pm 0.000033-3.2$

$0.280986 \pm 0.000050-2.4$

$0.282035 \pm 0.000056 \quad-2.4$

$0.281110 \pm 0.000093 \quad-26.7$

$0.282416 \pm 0.000056-27.9$

$0.282790 \pm 0.000069-13.1$

$0.282142 \pm 0.000025 \quad-8.9$

$0.282004 \pm 0.000069-42.3$

$0.282593 \pm 0.000037-16.7$
1067

$1889 \quad 2090$

$2882 \quad 3255$

$1771 \quad 2743$

$1742 \quad 1837$

$3426 \quad 3862$

$2008 \quad 2228$

$1475 \quad 1511$

$862 \quad 1353$

$1737 \quad 1737$

$1659 \quad 172$

$1240 \quad 1945$

$1419 \quad 1449$

$1871 \quad 1993$

$1787 \quad 1875$

$1313 \quad 1443$

$1670 \quad 1670$

$1331 \quad 1468$

$363 \quad 568$

$2979 \quad 3019$

$2693 \quad 2741$

$1483 \quad 1718$

$3080 \quad 3080$

$1201 \quad 1892$

$1540 \quad 1771$

$1675 \quad 1754$

$2871 \quad 2928$

$1563 \quad 1621$

$2920 \quad 3555$

$1306 \quad 2045$

$694 \quad 1049$

$1484 \quad 1708$

$1957 \quad 3058$

$979 \quad 1425$ 


\begin{tabular}{|c|c|c|c|c|c|c|c|c|c|c|c|c|c|}
\hline n404: & 0.082 & \pm 0.013 & $0.00406 \pm 0.00021$ & 26 & \pm & $0.282332 \pm 0.000084$ & -31.0 & 1488 & 2297 & $0.282332 \pm 0.000084$ & -31.1 & 1444 & 266 \\
\hline 05 & 0.097 & \pm 0.004 & $0.18943 \pm 0.00488$ & 1569 & \pm 71 & $0.281925 \pm 0.000082$ & -4.7 & 1784 & 1902 & $0.281923 \pm 0.000082$ & -3.2 & 1712 & 790 \\
\hline 06 & 0.069 & \pm 0.003 & $0.15639 \pm 0.00404$ & 895 & \pm 93 & $82273 \pm 0.000058$ & -10.4 & 75 & 41 & $272 \pm 0.000058$ & -9.6 & 4 & 569 \\
\hline nn407 & 0.096 & \pm 0.003 & $0.20499 \pm 0.00656$ & 1552 & \pm 65 & $22 \pm 0.000082$ & -5.3 & 791 & 923 & $81920 \pm 0.0$ & -3.8 & 720 & 813 \\
\hline & 0.078 & \pm 0.003 & $4 \pm 0.00375$ & 1151 & \pm 68 & & & & & & & & \\
\hline 09 & 0.217 & \pm 0.006 & $0.62399 \pm 0$ & 2955 & \pm 46 & $280926 \pm 0.000047$ & -2.3 & 3060 & 114 & $922 \pm 0.000047$ & 0.7 & 955 & 955 \\
\hline 10 & 0.063 & \pm 0.005 & $0.07103 \pm$ & 442 & \pm 18 & $0.282361 \pm 0.000048$ & -19.2 & 1341 & 1836 & $282360 \pm 0.000048$ & -18.8 & 294 & 785 \\
\hline 11 & 0.053 & \pm 0.017 & $0.17089 \pm 0.00809$ & 1017 & \pm 44 & $0.282013 \pm 0.000056$ & -16.3 & 1772 & 2181 & $.282012 \pm 0.000056$ & -15.4 & 705 & 092 \\
\hline 12 & 0.072 & \pm 0.006 & $0.18073 \pm 0.00789$ & 1071 & \pm 43 & $0.282163 \pm 0.000049$ & -9.6 & 1513 & 1757 & $0.282162 \pm 0.000049$ & -8.6 & 1457 & 674 \\
\hline 1413 & 0.077 & \pm 0.004 & $0.18978 \pm 0.00637$ & 1125 & \pm 102 & $0.282294 \pm 0.000063$ & -3.5 & 1287 & 1379 & $0.282291 \pm 0.000063$ & -2.5 & 1237 & 303 \\
\hline 1414 & 0.093 & \pm 0.002 & $0.24733 \pm 0.00936$ & 1487 & $\pm \quad 40$ & $0.281992 \pm 0.000134$ & -4.5 & 1693 & 1807 & $0.281991 \pm 0.000134$ & -3.1 & 1625 & 700 \\
\hline zrn415 & 0.068 & \pm 0.004 & $0.17387 \pm 0.00711$ & 859 & \pm 127 & $0.282069 \pm 0.000071$ & -18.5 & 1718 & 2185 & $0.282068 \pm 0.000071$ & -17.7 & 1655 & 2105 \\
\hline rn416 & 0.102 & \pm 0.005 & $0.24784 \pm 0.00973$ & 1656 & $\pm \quad 97$ & $0.281983 \pm 0.000061$ & -0.3 & 1669 & 1676 & $0.281980 \pm 0.000061$ & 1.2 & 1656 & 1656 \\
\hline \multicolumn{14}{|c|}{ The zircon grains which ${ }^{207} \mathrm{~Pb}$ analytical intensities ar } \\
\hline \multicolumn{14}{|c|}{ The siganl intensities of ${ }^{207} \mathrm{~Pb}$ are undetectable. } \\
\hline \multicolumn{14}{|c|}{$\S \mathrm{U}-\mathrm{Pb}$ age represents ${ }^{207} \mathrm{~Pb} /{ }^{206} \mathrm{~Pb}$ age for grains where analytical intensities of ${ }^{207} \mathrm{~Pb}$ are higher than 1,000 count per second, and ${ }^{206} \mathrm{~Pb} /{ }^{238} \mathrm{U}$ age for other grains. } \\
\hline \multicolumn{14}{|c|}{ **The decay constant of ${ }^{176} \mathrm{Lu}: 1.865 \times 10^{-11}\left(\mathrm{yr}^{-1}\right)\left(\right.$ Scherer et al., 2001) and $1.983 \times 10^{-11}\left(\mathrm{yr}^{-1}\right)$ (Bizzaro et al., 2003) } \\
\hline
\end{tabular}

